\title{
Internal structure of fluctuating Cooper pairs
}

\author{
N. Andrenacci ${ }^{1,2}$, M. Capezzali ${ }^{1,3, a}$, and H. Beck ${ }^{1}$ \\ 1 Institut de Physique, Université de Neuchâtel, 2000 Neuchâtel, Switzerland \\ 2 Dipartimento di Fisica, Universitá degli Studi di Salerno, 84084 Baronissi, Italy \\ 3 School of Engineering, Swiss Federal Institute of Technology (EPFL), 1015 Ecublens, Switzerland
}

Received 10 January 2006 / Received in final form 12 September 2006

Published online 1st November 2006 - C EDP Sciences, Società Italiana di Fisica, Springer-Verlag 2006

\begin{abstract}
In order to obtain information about the internal structure of fluctuating Cooper pairs in the pseudogap state and below the transition temperature of high $T_{c}$ superconductors, we solve the BetheSalpeter equation for the two-electron propagator in order to calculate a "pair structure function" $g_{P}(\mathbf{P}, \boldsymbol{\rho})$ that depends on the internal distance $\boldsymbol{\rho}$ between the partners and on the center of mass momentum $\mathbf{P}$ of the pair. We use an attractive Hubbard model with a local potential for s-wave and a separable potential for d-wave symmetry. The amplitude of $g_{P}$ for small $\rho$ depends on temperature, chemical potential and interaction symmetry, but the $\rho$ dependence itself is rather insensitive to the interaction strength. Asymptotically $g_{P}$ decreases as an inverse power of $\rho$ for weak coupling, but exponentially when a pseudogap develops for stronger interaction. Some possibilities of observing the pair structure experimentally are mentioned.
\end{abstract}

PACS. 74.20.Fg BCS theory and its development - 74.20.Rp Pairing symmetries (other than s-wave)

\section{Introduction}

High-temperature cuprate superconductors show remarkable deviations from Fermi liquid behaviour in their normal state, in particular in the underdoped region. One of the most striking features is the opening of a pseudogap above the superconducting critical temperature $T_{c}$ and below another temperature, $T^{*}$, that increases when the doping is reduced [1]. This pseudogap appears to have the same angular dependence and magnitude as the superconducting gap below $T_{c}$, with which it seems to merge at the phase transition. In the pseudogap region, thermodynamic quantities and transport coefficients also deviate from Fermi liquid behaviour [2]. To explain these anomalies, different scenarios can be invoked. One of them is based on the formation in the pseudogap region of incoherent Cooper pairs [3], also called "preformed" pairs [4]. The pairing in both the spin and charge channels could indeed explain some of the forementioned normal state anomalies [5]. The phase coherence among these pairs is established at a lower temperature, leading to the superconducting transition. In this respect, the superconducting transition can be regarded almost as a Bose condensation of preformed Cooper pairs [7], while the BCS behaviour, in which the formation of the Cooper pairs

\footnotetext{
a e-mail: massimiliano.capezzali@epfl.ch
}

and their condensation occur at the same temperature, is recovered as one approaches the overdoped region.

It is customary to characterize the physical behaviour of a superconductor in terms of specific length scales. The low temperature superconducting coherence length $\xi_{s c}$ is an important quantity showing different behaviour between the overdoped and underdoped region. Indeed, $\xi_{s c}$ is much shorter in underdoped compounds. In the framework of model calculations one finds a marked dependence of this coherence length on the strength of the pairing interaction [8]. When the latter increases the system crosses over from the BCS regime towards the Bose-Einstein condensation (BEC) region, while $\xi_{s c}$ decreases. In the framework of a Landau-Ginzburg description of superconductivity $\xi_{s c}$ is the length scale over which the superconducting order parameter varies, for example around a given disturbance.

Another basic length, $\xi_{\text {pair }}$, is usually related to the size of the Cooper pairs in the superconducting condensate. In the BCS limit it can be obtained in a simple manner from the modulus square of the anomalous Green function $[9,10]$. It turns out that the behaviour of the two important length scales, $\xi_{s c}$ and $\xi_{\text {pair }}$, are similar as a function of coupling strength: the stronger the interaction the shorter are these two lengths (at least up to some intermediate coupling strength). 
Our interest here is focused on fluctuating pairs which have a finite lifetime and whose phases are correlated over a finite distance only. Above $T_{c}$ they precisely correspond to the above mentioned preformed pairs, but they also exist below $T_{c}$ due to thermal and quantum fluctuations. We will show that these fluctuating pairs should be characterized by a third typical length $\xi_{f p}$ which is determined by the dynamic pair correlation function, and is thus not necessarily directly related to the two other lengths introduced above.

We base our calculations on the two-dimensional attractive Hubbard model. We consider either an isotropic attraction with varying range (going from an on-site to a long range attraction) giving rise predominantly to $s$-wave pairing, and, alternatively, an attraction acting on electrons sitting on neighboring sites, leading to d-wave pairing. Analytic $[7,11]$ and numerical work $[12,13]$ has shown that such a model can describe the instabilities toward the formation of electronic pairs of these two symmetries, and the transition to the superconducting state. By increasing the strength of the attraction, it is possible to follow the evolution of a variety of physical quantities in a cross-over between a BCS-like behavior to a scenario where superconductivity can be related to the condensation of preformed pairs [14]. Going beyond the weak coupling limit, the formation of a pseudogap above the transition temperature is found.

In order to describe the internal structure of preformed pairs, for both $T$ larger than $T_{c}$ and $T$ smaller than $T_{c}$, in underdoped high-temperature superconductors we study the Green function for the operator which creates a pair of particles whose partners are at a certain relative distance, and study how this internal distance varies as a function of temperature, interaction strength and form, as well as density of particles. To this end, we will consider a Bethe-Salpeter (BS) approach. In the case of a dilute system of charge carriers, which is of interest to us, the simplest approximation to the BS equation allows to separate the center of mass motion of a pair from its internal degree of freedom. The former is described by the standard two-particle T-matrix. The latter takes into account the relevant interaction channel of repeated twoparticle scattering and has a two-fold meaning. On one hand, it correspond to the propagator of a pair with center of mass momentum $\mathbf{P}$, but having a specific internal structure, given by the form of the basic attractive interaction. On the other hand, the same T-matrix also has essentially the same form as the dynamic correlation function of the pairing field, which is governed by a linear approximation to the usual Landau-Ginzburg (LG) type free energy, that can be found from the basic Hamiltonian by the wellknown Stratonovich-Hubbard transformation [11]. In the framework of this LG description one immediately finds $\xi_{s c}$, which is expressed in the usual way by the coefficients of the Landau-Ginzburg free energy [8].

As stated above, we are, however, interested in the behaviour of the pair propagator for arbitrary distance between the two partners of the pair. This information comes from the two-particle bubble showing up in the BS equation, and it is finally given in the form of a pair structure function $g_{P}(\rho)$ which gives essentially the probability for the two partners of a pair, with a given center of mass momentum $\mathbf{P}$, of being at the distance $\boldsymbol{\rho}$ from each other. Interesting information about the internal structure of a pair can be obtained from the $\boldsymbol{\rho}$ dependence of this distribution function both for small and for large values of $\boldsymbol{\rho}$. The structure of $g_{P}(\rho)$ up to an internal distance of a few lattice constants, determined numerically, allows to exhibit the influence of various parameters, such as temperature, coupling strength, center of mass momentum and pairing symmetry. The asymptotic behaviour for large $\rho$ will be studied both numerically and analytically. For very weak coupling $g_{P}(\rho)$ goes to zero as a power of the distance, such that the second moment which is usually used in order to define $\xi_{\text {pair }}$ does not exist. On the other hand the existence of a pseudogap leads to an exponential decay. Thus, in this latter case a fluctuating pair length $\xi_{f p}$ can be introduced. Its value approaches the one of the length $\xi_{\text {pair }}$ of condensed Cooper pairs when the pairing interaction becomes stronger. This is a sign that towards strong coupling the superconducting transition can more and more be interpreted as the Bose condensation of the preformed pairs.

The paper is organized as follows. An overview of our theoretical approach is given in Section 2. Starting from the BS equation for both, $T$ larger than $T_{c}$ and $T$ smaller than $T_{c}$, an explicit expression is derived for $g_{P}(\rho)$, and the basic lengths are related in detail to the relevant quantities obtained from the BS equation. Above $T_{c}$ the one-electron Green functions constituting the two-particle bubble will either be approximated by a free electron form or by a BCS form with a finite line-width, in order to represent the pseudogap. In Section 3 we analyze the dependence of $g_{P}(\rho)$ on the distance $\boldsymbol{\rho}$ between the two partners of a pair. The short range structure reflects the underlying ( $\mathrm{s}$ or $\mathrm{d}$ ) symmetry of the pairing mechanism. Most pairs are relatively localized, extending only over a few lattice constants. Asymptotically $g_{P}(\rho)$ varies as an inverse power law of $\boldsymbol{\rho}$ for weak attraction, but exponentially when the interaction is strong enough to produce a pseudogap in the one-electron spectrum. In that case a characteristic length $\xi_{f p}$ of fluctuating pairs can be introduced and compared to $\xi_{\text {pair }}$ of the superconducting state. We then discuss the influence of the various parameters of our model, such as temperature, chemical potential and interaction strength and shape, on the structure of $g_{P}(\rho)$. Pairs formed on top of the Fermi sea remain relatively localized even when the coupling becomes weak. The main results of our work are summarized in Section 4, where we also mention possible experimental accesses to the internal structure of Cooper pairs.

In Appendix A we discuss the form of the oneelectron spectral functions in the pseudogap region and in Appendix B we relate our pair structure function to the total number of preformed pairs which is usually introduced in the framework of the Landau-Ginzburg description of the superconducting phase transition. In 
Appendix $\mathrm{C}$ we discuss the pair length for a d-wave superconductor at finite temperature.

\section{Theoretical description of the pairs}

We consider an extended attractive two-dimensional Hubbard model given by the Hamiltonian:

$$
\begin{aligned}
H= & -t \sum_{\langle i, j\rangle, \sigma}\left(c_{i \sigma}^{\dagger} c_{j \sigma}+\text { h.c. }\right)-\sum_{i, j} U(i-j) n_{i \downarrow} n_{j \uparrow} \\
& -\mu \sum_{i}\left(n_{i \downarrow}+n_{i \uparrow}\right)
\end{aligned}
$$

where $t$ is the hopping parameter between nearest neighbor sites and $U(i-j)>0$ is an attractive interaction coupling the electronic density $n_{i \sigma}=c_{i \sigma}^{\dagger} c_{i \sigma}$ at two different sites $i$ and $j$. All our calculations are performed in the grand-canonical ensemble, using the chemical potential $\mu$, rather than the electronic density, as a parameter. Our objective consists in analyzing, in the framework of (1), the structure of electronic pairs. The latter shows up in different context. There are "preformed" or "fluctuating" pairs in the normal state for $T>T_{c}$, in the domain where simulations show the formation of a pseudogap. One of the current interpretations of this unusual feature in the cuprates precisely invokes the presence of preformed electronic pairs, existing up to a temperature $T^{*}$, that can be substantially higher than $T_{c}$. Below $T_{c}$, Cooper pairs form the superconducting condensate, but there are also "fluctuating" pairs in the superconducting state. In order to describe this broken symmetry phase, we will introduce the Nambu formalism. In this way, we create a formalism that is valid above and below $T_{c}$.

In order to study electronic pairs we consider the operator

$$
Q^{\dagger}\left(\mathbf{r}_{i j}, \boldsymbol{\rho}_{i j}\right)=c_{i \uparrow}^{\dagger} c_{j \downarrow}^{\dagger},
$$

defined on the lattice sites $i$ and $j$ with coordinates $\mathbf{x}_{i}$ and $\mathbf{x}_{j}$, which creates a pair of electrons with opposite spins. We define the center of mass coordinate of the pair $\mathbf{r}_{i j}$ and the relative distance $\boldsymbol{\rho}_{i j}$, as:

$$
\begin{aligned}
\mathbf{r}_{i j} & =\frac{1}{2}\left(\mathbf{x}_{i}+\mathbf{x}_{j}\right) \\
\boldsymbol{\rho}_{i j} & =\mathbf{x}_{i}-\mathbf{x}_{j} .
\end{aligned}
$$

Although $Q^{\dagger}$ and its adjoint do not satisfy the canonical commutation relations for independent (bosonic) particles, they can be used in order to describe electronic pair correlations through the dynamic correlation function:

$$
\begin{aligned}
\chi\left(\mathbf{r}_{i j}, \boldsymbol{\rho}_{i j}, t ; \mathbf{r}_{r s}, \boldsymbol{\rho}_{r s}, t^{\prime}\right) & =(-i) \\
\times & \left\langle T\left[Q\left(\mathbf{r}_{i j}, \boldsymbol{\rho}_{i j}, t\right) Q^{\dagger}\left(\mathbf{r}_{r s}, \boldsymbol{\rho}_{r s}, t^{\prime}\right)\right]\right\rangle,
\end{aligned}
$$

where $T$ denotes the time ordering operator. Manifestly, $\chi$ is a two-electron Green function $G_{2}$ :

$$
G_{2}\left(1,2 ; 1^{\prime}, 2^{\prime}\right)=-\left\langle T\left[c(1) c(2) c^{\dagger}\left(2^{\prime}\right) c^{\dagger}\left(1^{\prime}\right)\right]\right\rangle,
$$

with a special choice of arguments (the numbers $i=$ $1,2,1^{\prime}, 2^{\prime}$ are a shorthand notation for the generalized coordinates $\mathbf{x}_{i}, t_{i}, \sigma_{i}$ of space, time and spin variables):

$$
\chi\left(\mathbf{r}_{i j}, \boldsymbol{\rho}_{i j}, t ; \mathbf{r}_{r s}, \boldsymbol{\rho}_{r s}, t^{\prime}\right)=i G_{2}\left(\mathbf{x}_{i}, t ; \mathbf{x}_{j}, t ; \mathbf{x}_{r}, t^{\prime} ; \mathbf{x}_{s}, t^{\prime}\right) .
$$

A simple and convenient approximation for evaluating $G_{2}$ for a system described by the Hamiltonian (1) consists in solving the Bethe-Salpeter (BS) equation [15], which we study separately in the normal and in the superconducting states.

\subsection{Normal state}

Here the two-electron Green function has the form:

$$
\begin{aligned}
G_{2}\left(1,2 ; 1^{\prime}, 2^{\prime}\right) & =G\left(1,1^{\prime}\right) G\left(2,2^{\prime}\right)-G\left(1,2^{\prime}\right) G\left(2,1^{\prime}\right) \\
& +i G(1, \underline{1}) G(2, \underline{2}) U(\underline{1}-\underline{2}) G_{2}(1,2 ; \underline{1}, \underline{2})
\end{aligned}
$$

taking into account only repeated scattering events between two electrons (thus restricting our analysis to low density systems) and expressing $G_{2}$ in term of the "full" one-electron Green's function $G$ and of the attraction potential $U$. Here and in the following we use imaginary time arguments, $0 \leq t \leq i \beta, \beta=1 /\left(k_{B} T\right)$, with the corresponding time-ordering. We also adopted in (6) the convention for which the bar under two identical arguments denotes integration over space and time. Introducing the special choice of variables needed for the pair correlation (5) into the general BS equation (6) and denoting the dynamic correlation function (3) by $\chi\left(\mathbf{r}_{i j}-\mathbf{r}_{r s}, \boldsymbol{\rho}_{i j}, \boldsymbol{\rho}_{r s}, t-t^{\prime}\right) \equiv$ $\chi\left(\mathbf{r}_{i}, \boldsymbol{\rho}_{j}, \boldsymbol{\rho}_{l}, t\right)$ (where now $\mathbf{r}_{i}=\mathbf{i} a$ and $\boldsymbol{\rho}_{j}=\mathbf{j} a$ are discrete vectors, that represent the distance among two sites on a square lattice of constant $a$ ), one obtains:

$$
\begin{aligned}
\chi\left(\mathbf{r}_{i}, \boldsymbol{\rho}_{j}, \boldsymbol{\rho}_{l}, t\right) & =-i \mathcal{G}\left(\mathbf{r}_{i}, \boldsymbol{\rho}_{j}-\boldsymbol{\rho}_{l}, t\right)+i \int d t^{\prime} \\
& \times \sum_{m} \sum_{k} \mathcal{G}\left(\mathbf{r}_{i}-\mathbf{r}_{m}, \boldsymbol{\rho}_{j}-\mathbf{r}_{k}, t-t^{\prime}\right) U\left(\mathbf{r}_{k}\right) \\
& \times \chi\left(\mathbf{r}_{m}, \mathbf{r}_{k}, \boldsymbol{\rho}_{l}, t^{\prime}\right)
\end{aligned}
$$

where $\mathcal{G}$ is the particle-particle bubble:

$$
\mathcal{G}\left(\mathbf{r}_{i}, \boldsymbol{\rho}_{j}, t\right)=G\left(\mathbf{r}_{i}+\frac{\boldsymbol{\rho}_{j}}{2}, t\right) G\left(\mathbf{r}_{i}-\frac{\boldsymbol{\rho}_{j}}{2}, t\right),
$$

describing the motion of two independent particles whose properties have been renormalized by the interaction. The spin indices have been omitted, since we are interested in the particular configuration of opposite spin of the partners of the pairs, and the interaction is independent of the spin variable. We now apply a Fourier transformation with respect to the variable $\mathbf{r}_{i}$ to equation (7), introducing the center of mass momentum $\mathbf{P}$, and we go from imaginary time to (bosonic) Matsubara frequencies $z_{\nu}$. For a local attraction $U(\mathbf{r})=U_{0} \delta(\mathbf{r})$ the transformed equation becomes algebraic and $\chi$ can immediately be related to the particle-particle bubble function $\mathcal{G}$ (see below). When the interaction has a finite spatial extension one has either to 
solve numerically the integral equation (7) or to introduce a separable form for the Fourier transform of $U(i-j)$ :

$$
\begin{aligned}
\tilde{U}\left(\mathbf{k}-\mathbf{k}^{\prime}\right) & \equiv \sum_{l} e^{i\left(\mathbf{k}-\mathbf{k}^{\prime}\right) \cdot \mathbf{x}_{l}} U\left(\mathbf{x}_{l}\right) \\
& =\sum_{\lambda} U_{\lambda} \phi_{\lambda}(\mathbf{k}) \phi_{\lambda}^{*}(\mathbf{k}),
\end{aligned}
$$

$\phi_{\lambda}$ being the various characteristic shape functions of the potential. In order to simplify the formalism we will select one value of $\phi_{\lambda}$ only. We will consider the following cases:

$$
\begin{gathered}
\phi_{\text {iso }}(\mathbf{k})=\frac{1}{\sqrt{1+k^{2} / k_{0}^{2}}} \\
\phi_{d}(\mathbf{k})=\cos k_{x} a-\cos k_{y} a .
\end{gathered}
$$

The first weight function is the usual form for an isotropic interaction [9] with a typical interaction range given by the inverse of $k_{0}$. For $k_{0} \rightarrow \infty$ the usual point interaction leading to s-wave pairing is recovered. The second choice is for $\mathrm{d}$-wave pairing. The equation for $\chi$ is thus given by:

$$
\begin{aligned}
\chi\left(\mathbf{P}, \boldsymbol{\rho}, \boldsymbol{\rho}^{\prime}, z_{\nu}\right)= & -i \sum_{\mathbf{k}} e^{-i \mathbf{k}\left(\rho-\rho^{\prime}\right)} \tilde{\mathcal{G}}\left(\mathbf{P}, \mathbf{k} ; z_{\nu}\right)+i \sum_{\lambda} U_{\lambda} \\
& \times \sum_{\mathbf{k}} e^{-i \mathbf{k} \cdot \boldsymbol{\rho}} \phi_{\lambda}^{*}(\mathbf{k}) \tilde{\mathcal{G}}\left(\mathbf{P}, \mathbf{k} ; z_{\nu}\right) \\
& \times \sum_{\mathbf{k}^{\prime}} e^{-i \mathbf{k}^{\prime} \cdot \boldsymbol{\rho}^{\prime}} F_{\lambda}\left(\mathbf{P}, \mathbf{k} ; z_{\nu}\right),
\end{aligned}
$$

where $\tilde{\mathcal{G}}\left(\mathbf{P}, \mathbf{k} ; z_{\nu}\right)$ is the Fourier transform of the particleparticle bubble (8) with respect to the center of mass position $\mathbf{r}$, internal distance $\boldsymbol{\rho}$ and time, and $F_{\lambda}$ is an intermediate-step quantity obtained from the convolution of the Fourier transform of the dynamic correlation function $\chi$ with respect to the variables $\boldsymbol{\rho}_{i}$ and $\boldsymbol{\rho}_{j}$, and the characteristic shape function of the potential:

$$
\begin{aligned}
F_{\lambda}\left(\mathbf{P}, \mathbf{k} ; z_{\nu}\right)= & \sum_{\mathbf{k}^{\prime}} \phi_{\lambda}\left(\mathbf{k}^{\prime}\right) \sum_{i} \sum_{j} e^{i \mathbf{k} \cdot \boldsymbol{\rho}_{i}} e^{-i \mathbf{k}^{\prime} \cdot \boldsymbol{\rho}_{j}} \\
& \times \chi\left(\mathbf{P}, \boldsymbol{\rho}_{i}, \boldsymbol{\rho}_{j}, z_{\nu}\right) .
\end{aligned}
$$

For a given weight function in (9) the equations (12) and (13) can now be solved to yield:

$$
\begin{aligned}
\chi\left(\mathbf{P}, \boldsymbol{\rho}, \boldsymbol{\rho}, z_{\nu}\right)= & -i \mathcal{G}\left(\mathbf{P}, \mathbf{0}, z_{\nu}\right)+T\left(\mathbf{P}, z_{\nu}\right) \\
& \times \hat{\mathcal{G}}\left(\mathbf{P}, \boldsymbol{\rho}, z_{\nu}\right) \hat{\mathcal{G}}\left(\mathbf{P},-\boldsymbol{\rho}, z_{\nu}\right) .
\end{aligned}
$$

Here the following key quantities show up:

1. The usual two-particle T-matrix $T\left(\mathbf{P}, z_{\nu}\right)$ given by:

$$
T\left(\mathbf{P}, z_{\nu}\right)=\frac{U_{\lambda}}{1-i U_{\lambda} \sum_{\mathbf{k}}\left|\phi_{\lambda}(\mathbf{k})\right|^{2} \tilde{\mathcal{G}}\left(\mathbf{P}, \mathbf{k} ; z_{\nu}\right)} .
$$

Given the fact that it involves - in the denominator - a sum over the wave vector $\mathbf{k}$ of the particle-particle bubble, weighted by the square of the shape function determining the interaction potential, it contains information about a particular type of "reference pairs" with a given internal structure (determined precisely by the square of the weight function) with center of mass momentum $\mathbf{P}$, and Matsubara frequency $z_{\nu}$ corresponding to the energy of the pair when continued to the real axis. For a strictly local potential (i.e. $\phi_{\lambda}=1$ ) the corresponding reference pairs consist of electrons sitting on the same lattice site. As usual the T-matrix, for $z_{\nu}$ going to $\Omega+i \epsilon$, can be given a simple form for small $\Omega$ and $\mathbf{P}$ :

$$
T(\mathbf{P}, \Omega)=\left[a+c \boldsymbol{P}^{2}+d \Omega\right]^{-1} .
$$

This form corresponds to the dynamic correlation function of the pairing field $\psi$ governed by a time-dependent Landau-Ginzburg equation:

$$
d \frac{\partial \psi}{\partial t}=-a \psi+c \nabla^{2} \psi
$$

Indeed, starting from the above Hamiltonian with a separable potential of the form (9) and upon performing the usual Stratonovich-Hubbard transformation [11], one can transform the partition function into a functional integral over such a time- and space-dependent pairing field $\psi$, governed by a Landau-Ginzburg-type free energy, which is quadratic in $\psi$ and leads to the field equation (56). In this framework the typical length scale characterizing the "stiffness" of the pairing field can be identified, which is our superconducting coherence length $\xi_{s c}$. The above coefficient $a$ usually has the following temperature dependence

$$
a(T)=a_{0}\left(1-\frac{T}{T_{c}}\right) .
$$

Based on this form, Kao et al. [8] define $\xi_{s c}$ by:

$$
\xi_{s c}^{2}=c / a_{0}
$$

They note that this length strongly depends on the strength of the interaction: it is large for weak coupling and it becomes small for intermediate coupling.

2. The two-particle bubble, Fourier transformed back to real space by using the weight factor $\phi_{\lambda}$, reads:

$$
\hat{\mathcal{G}}\left(\mathbf{P}, \boldsymbol{\rho}, z_{\nu}\right)=\sum_{k} e^{i \mathbf{k} \cdot \boldsymbol{\rho}} \phi_{\lambda}(\mathbf{k}) \tilde{\mathcal{G}}\left(\mathbf{P}, \mathbf{k}, z_{\nu}\right) .
$$

This is the quantity which introduces the $\boldsymbol{\rho}$ dependence into $\chi$ which we are actually interested in. In order to interpret in simple physical terms the internal structure of the preformed pairs which we will find in the following section we will thus have to come back to the $\boldsymbol{\rho}$ dependence hidden in (20).

Finally, the "pair structure function" $g_{P}(\mathbf{P}, \boldsymbol{\rho})$, that gives the internal structure of a pair having momentum $\mathbf{P}$ and internal distance $\rho$ is defined by:

$$
g_{P}(\mathbf{P}, \boldsymbol{\rho})=\int d \Omega N_{B}(\Omega) \Phi(\mathbf{P}, \boldsymbol{\rho}, \Omega),
$$

$N_{B}(\Omega)$ being the Bose-Einstein distribution function, while $\Phi(\mathbf{P}, \boldsymbol{\rho}, \Omega)$ represents the spectral function of a pair 
with center of mass momentum $\mathbf{P}$, energy $\Omega$ and internal distance $\boldsymbol{\rho}$ :

$$
\Phi(\mathbf{P}, \boldsymbol{\rho}, \Omega)=\operatorname{Im}\left\{\chi\left(\mathbf{P}, \boldsymbol{\rho}, \boldsymbol{\rho}, \Omega+i 0^{+}\right)\right\} .
$$

The form of the pair interaction influences $g_{P}(\rho)$ in two different ways:

a) It renormalizes the one-electron Green function constituting the bubble (20), in particular by creating a pseudogap in the density of states opening at a temperature $T^{*}$, which can be substantially higher than $T_{c}$

b) it shows up explicitly in the T-matrix expression (15) determing thus the coefficient $a(T)$ in (18) and thus the superconducting transition temperature (given in our approach by the so-called Thouless criterion, i.e. by the temperature at which $a(T)$ vanishes). It will thus also influence the coherence length $\xi_{s c}$, as stated above in (19). One has, however, to stress that the potential strength does not show up explicitly in the expression of the bubble which determines the internal structure of our preformed pairs. It is thus not surprising that - as we will discuss in Section 3 the influence of the potential strength on the distribution function $g_{P}(\mathbf{P}, \boldsymbol{\rho})$ of the pair size is weaker than its influence on quantities like $\xi_{s c}$.

Following expression (21) we can construct a function, $g_{P}^{0}(\mathbf{P}, \boldsymbol{\rho})$, that represents the internal structure of two independent particles with center of mass momentum $\mathbf{P}$ and which is given by the first term in (14):

$$
\begin{aligned}
g_{P}^{0}(\mathbf{P}, \boldsymbol{\rho}) & \equiv \int d \Omega N_{B}(\Omega) \operatorname{Im}\left\{\left.\chi^{0}\left(\mathbf{P}, \boldsymbol{\rho}, \boldsymbol{\rho}, z_{\nu}\right)\right|_{z_{\nu} \rightarrow \Omega+i 0^{+}}\right\} \\
& \equiv \int d \Omega N_{B}(\Omega) \operatorname{Im}\left\{\left.\mathcal{G}\left(\mathbf{P}, \mathbf{0}, z_{\nu}\right)\right|_{z_{\nu} \rightarrow \Omega+i 0^{+}}\right\}
\end{aligned}
$$

Being independent of $\boldsymbol{\rho}$, this contribution forms a constant "background" to the structure of $g_{P}(\rho)$, resulting from the direct two-particles scattering. For this reason, we subtract this background contribution from $g_{P}(\mathbf{P}, \boldsymbol{\rho})$, so that instead of the definition (22) we will use the "rescaled":

$$
\Phi(\mathbf{P}, \boldsymbol{\rho}, \Omega)=\operatorname{Im}\left\{\chi(\mathbf{P}, \boldsymbol{\rho}, \boldsymbol{\rho}, \Omega+i \eta)-\chi^{0}(\mathbf{P}, 0, \Omega+i \eta)\right\} .
$$

However, one has to bear in mind that possible negative values of $g_{P}(\mathbf{P}, \boldsymbol{\rho})$ simply mean a reduction of the number of corresponding pairs with respect to this independent particle background.

We do not attempt a fully self-consistent calculation of $G$ - and thus of $\mathcal{G}$ - and $\chi$. Rather, we approximate the one-electron spectral function in the pseudogap regime we are interested in by a BCS-like form with a finite linewidth $\gamma$. The corresponding result for $\mathcal{G}$ is derived in Appendix A.

\subsection{Superconducting state}

In this domain we deal with fluctuating pairs which still exist below $T_{c}$. The formal procedure follows the same lines as above $T_{c}$, but it is slightly more complicated due to the existence of the anomalous one-particle Green functions. The BS approach has already been used to study the Cooper pairs in the BCS ground state for an ideal Fermi gas [6]. The two particle propagator has two poles that represent a massless mode (the Anderson-BogoliubovHiggs mode) and a massive mode, describing pairs that are two-particle bound states with a finite lifetime, as the "preformed" pairs above $T_{c}$. It is useful to introduce the Nambu representation for the particle field operator:

$$
\Psi(\mathbf{r})=\left(\begin{array}{c}
\psi_{\uparrow}(\mathbf{r}) \\
\psi_{\downarrow}^{\dagger}(\mathbf{r})
\end{array}\right)
$$

so that the BCS Green functions can be written as:

$$
\mathbf{G}(1,2)=-\left\langle T\left[\Psi(1) \Psi^{\dagger}(2)\right]\right\rangle
$$

and the two-particle Green's function:

$$
\mathbf{G}_{2}\left(1,2 ; 1^{\prime}, 2^{\prime}\right)=\left\langle T\left[\Psi(1) \Psi(2) \Psi^{\dagger}\left(2^{\prime}\right) \Psi^{\dagger}\left(1^{\prime}\right)\right]\right\rangle
$$

where the argument of the field operator represents the coordinates and the index of the Nambu spinor component $1=\mathbf{r}_{1}, \tau_{1}, \ell_{1}$, etc. In order to solve a BS equation analogous to (6) it is convenient to introduce a reduced two-particle correlation function:

$$
L\left(1,2 ; 1^{\prime}, 2^{\prime}\right)=G_{2}\left(1,2 ; 1^{\prime}, 2^{\prime}\right)-G\left(1,1^{\prime}\right) G\left(2,2^{\prime}\right)
$$

which satisfies the BS equation:

$$
\begin{aligned}
L\left(1,2 ; 1^{\prime}, 2^{\prime}\right)= & -G\left(1,2^{\prime}\right) G\left(2,1^{\prime}\right)+G(1, \underline{1}) G\left(\underline{4}, 1^{\prime}\right) \\
& \times \mathcal{U}(\underline{1}, \underline{3} ; \underline{4}, \underline{2}) L\left(\underline{2}, 2 ; \underline{3}, 2^{\prime}\right)
\end{aligned}
$$

where $\mathcal{U}$ is an effective two particle interaction that depends on the Nambu indices as well as on the particles space and time coordinates. The above equation can be solved introducing a many-particle T-matrix:

$$
\begin{aligned}
T\left(1,2 ; 1^{\prime}, 2^{\prime}\right)= & \mathcal{U}\left(1,2 ; 1^{\prime}, 2^{\prime}\right)+\mathcal{U}\left(1, \underline{2} ; 1^{\prime}, \underline{1}\right) \\
& \times G(\underline{1}, \underline{4}) G(\underline{3}, \underline{2}) T\left(\underline{4}, 2 ; \underline{3}, 2^{\prime}\right)
\end{aligned}
$$

and by expressing $L$ in function of $T$ :

$$
\begin{aligned}
-L\left(1,2 ; 1^{\prime}, 2^{\prime}\right)= & G\left(1,2^{\prime}\right) G\left(2,1^{\prime}\right)+G(1, \underline{1}) G\left(\underline{4}, 1^{\prime}\right) \\
& \times T(\underline{1}, \underline{3} ; \underline{4}, \underline{2}) G\left(\underline{2}, 2^{\prime}\right) G(2, \underline{3}) .
\end{aligned}
$$

For the choice of the space and time coordinates made in equation (5), the corresponding two-particle correlation function in Nambu representation is given by:

$$
\begin{aligned}
L\left(1,2 ; 1^{\prime}, 2^{\prime}\right) & =L\left(\mathbf{x}_{j}, t ; \mathbf{x}_{r}, t^{\prime} ; \mathbf{x}_{i}, t ; \mathbf{x}_{s}, t^{\prime}\right)_{1221} \\
& \equiv L\left(x_{1} ; x_{1}^{\prime} ; x_{1} ; x_{2^{\prime}}\right)_{1221}
\end{aligned}
$$

and the two particle Green's function, for the arguments we are interested in, is given in Nambu representation (27) by:

$$
\begin{aligned}
G_{2}\left(x_{2} ; x_{1}^{\prime} ; x_{1} ; x_{2}^{\prime}\right)_{1221}= & G\left(x_{2} ; x_{1}\right)_{12} G\left(x_{1}^{\prime} ; x_{2}^{\prime}\right)_{21} \\
& +L\left(x_{2} ; x_{1}^{\prime} ; x_{1} ; x_{2}^{\prime}\right)_{1221}
\end{aligned}
$$


where $G(x, y)_{1,2}=G(x, y)_{2,1}^{*}$ is the anomalous BCS Green function.

This expression shows that in the superconducting state the pair correlation function has two contributions. The first term, involving the product of the two anomalous Green functions, represents the internal structure of pairs that belong to the superconducting condensate. Indeed, the anomalous Green functions depend only on the relative distance $\boldsymbol{\rho}$ among the partners and not on their centre of mass coordinate. Thus, their centre of mass momentum is zero. It is from this anomalous bubble that one usually extracts the pair length $\xi_{\text {pair }}$, which represents the average distance among fermions in a Cooper pair (see Appendix C). The second contribution to the pair correlation function (32), gives information about the non-condensed (or "fluctuating") pairs, as above the critical temperature. The method to calculate this part, i.e. to solve equation (30), is the same used for the pseudogap state. However, in the broken symmetry phase the longitudinal and the transverse components with respect to the direction of broken symmetry of the two particle correlation function are not equal (as it is instead the case above $T_{c}$ ). The information about the massless mode is carried by the tranverse part, which describes phase correlations among pairs, while the longitudinal component corresponds to the propagator of pairs with a given mass, and contains information about the relevant length of the broken symmetry phase $[21,22]$. In order to obtain information about the internal structure of this fluctuating pairs, we use the longitudinal part of the correlation function, given by [21]:

$$
\begin{aligned}
\chi_{\|}\left(x_{2}, x_{1}^{\prime} ; x_{1}, x_{2}^{\prime}\right)= & L\left(x_{2}, x_{1}^{\prime} ; x_{1}, x_{2}^{\prime}\right)_{1221} \\
& +L\left(x_{2}, x_{1}^{\prime} ; x_{1}, x_{2}^{\prime}\right)_{2112} \\
& +L\left(x_{2}, x_{1}^{\prime} ; x_{1}, x_{2}^{\prime}\right)_{2211} \\
& +L\left(x_{2}, x_{1}^{\prime} ; x_{1}, x_{2}^{\prime}\right)_{1122} .
\end{aligned}
$$

From the longitudinal pair correlation function we can define the pair structure function (21) below $T_{c}$ with:

$$
\Phi(\mathbf{P}, \boldsymbol{\rho}, \Omega)=\operatorname{Im}\left[\chi_{\|}(\mathbf{P}, \boldsymbol{\rho}, \boldsymbol{\rho}, \Omega+i \eta)-\chi_{\|}^{0}(\mathbf{P}, 0, \Omega+i \eta)\right]
$$

where $\chi_{\|}^{0}$ is the contribution of the bare bubbles to (33), due to the single particle Green functions product appearing in the definition of the two-particles correlation function (30). $\boldsymbol{\rho}$ and $\mathbf{P}$ are as for $T>T_{c}$. Following reference [23], we distinguish two gap parameters in the BCS Green function: the gap $\Delta_{p g}(\mathbf{k})$, which represents the pseudogap that survives below $T_{c}$, and the gap occurring in the anomalous Green function $\Delta_{s c}(\mathbf{k})$, which represents the finite average value of the pairing field, the order parameter in the broken symmetry phase. The quasi-particle dispersion can be written then as:

$$
E(\mathbf{k})=\sqrt{\xi(\mathbf{k})^{2}+\Delta(\mathbf{k})^{2}}
$$

where $\Delta(\mathbf{k})^{2} \equiv \Delta_{p g}(\mathbf{k})^{2}+\Delta_{s c}(\mathbf{k})^{2}$ and $\xi(\mathbf{k})=\epsilon(\mathbf{k})-\mu$ is the free particle dispersion. The Green functions can be written explicitly as:

$$
\begin{aligned}
& G\left(\mathbf{k}, \omega_{n}\right)_{11}=-\frac{\xi(\mathbf{k})+i \omega_{n}}{E(\mathbf{k})^{2}+\omega_{n}^{2}}=-G\left(-\mathbf{k},-\omega_{n}\right)_{22} \\
& G\left(\mathbf{k}, \omega_{n}\right)_{21}=-\frac{\phi(\mathbf{k})}{E(\mathbf{k})^{2}+\omega_{n}^{2}}=G\left(\mathbf{k}, \omega_{n}\right)_{12}
\end{aligned}
$$

\section{Numerical results}

Our numerical calculations are based on the tight-binding spectrum resulting from the kinetic energy part of the Hamiltonian (1) with a nearest neighbor hopping $t$ in two dimensions. We use the total bandwidth $W=8 t$ as our energy unit. The zero of the energy is in the center of the band. Assuming that the underdoped cuprates with a pronounced pseudogap are situated in an intermediate coupling regime of the attractive Hubbard model [16] we chose an interaction strength $U$ of the order of $0.5 \mathrm{~W}$. In the d-wave case, however, the interaction strength should be of order of the bandwidth to obtain reasonable values for the critical temperature $[17,18]$. The value of the gap at zero temperature has been calculated solving the BCS equation, for fixed value of the chemical potential.

In order to incorporate the effect of the finite lifetime of the pair excitations without washing out the effect of the pseudogap, we choose one-electron Green functions with a finite linewidth $\Gamma$ (see Appendix A) of the order of $\Delta / 2$, for which rather sharp features in the pseudogap region are still present.

For the width of the pseudogap we rely on quantum Monte Carlo calculations for the two-dimensional attractive Hubbard model [13]. Those results show that the value of the width depends on the band filling (i.e. on the chemical potential), but it is essentially independent of temperature $T$. The latter fact is one of the characteristic features of the pseudogap: when $T$ increases, above $T_{c}$, the pseudogap is filling up rather than closing. This filling up of the pseudogap can be taken into account by choosing a $T$-dependent line width $\Gamma$. For our interaction strength $(U=0.5 \mathrm{~W})$ the numerical value of the pseudogap halfwidth $\Delta_{p g}$ is on the order of $0.05 \mathrm{~W}$ for a quarter-filled band and to $0.06 \mathrm{~W}$ closer to half-filling. Our chemical potential values correspond to lower filling. We will therefore chose values below $0.05 \mathrm{~W}$ for $\Delta_{p g}$ in our calculations. The linewidth $\Gamma$ is then adapted in order to obtain one-electron spetral functions of the shape as in the simulations, i.e. with a rounded pseudogap, rather than a true gap.

The relevant temperature interval of the pseudogap regime is determined in the framework of our description of pairing. In the usual T-matrix approximation, which results from the BS equation (6), the superconducting instability occurs at the temperature where the "Thouless criterion" is fulfilled:

$$
T^{-1}\left(\mathbf{P}=0, z_{\nu}=0\right)=0,
$$

i.e., where pairs with zero centre of mass momentum begin to form a true bound state. This is a "mean-field-like" criterion that corresponds to our approach to pair structure, 
(a)

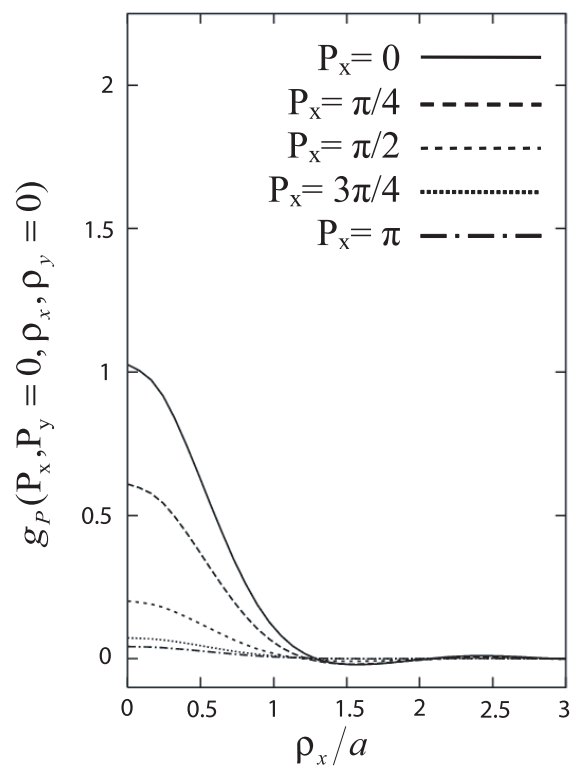

(b)

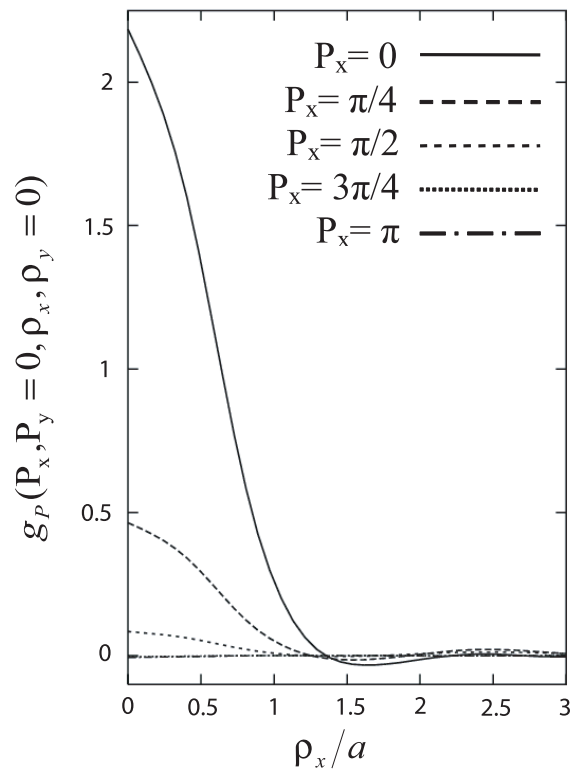

Fig. 1. Short range behaviour of the pair structure function $g_{P}(\mathbf{P}, \boldsymbol{\rho})$ as a function of $\boldsymbol{\rho}=\left(\boldsymbol{\rho}_{x}, 0\right)$ (in units of a) and $\mathbf{P}=\left(\mathbf{P}_{x}, 0\right)$. The relevant parameters are, respectively, $\mu=-0.4 W$, $U=-0.5 W$. Moreover for (a) $\Delta_{p g}=0$ and (b) $\Delta_{p g}=0.04 \mathrm{~W}$, and the ratio $T / T_{c}$ equals 1.5 for both figures. which is, of course, less precise than a treatment going more deeply into the analysis of phase fluctuations in the framework of the Kosterlitz-Thouless transition. For our calculations, we will in general indicate the value of the temperature ratio $T / T_{c}$, in order to compare similar situations for different values of the underlying parameters.

The imaginary part of the T-matrix $T\left(\mathbf{P}, \Omega+i 0^{+}\right)$is the spectral function of fluctuating pairs of a given internal structure, specified by the denominator of expression (16). The inverse width of $\operatorname{Im} T$ as a function of $W$ can be interpreted as the life time of the corresponding pair. We obtain, in the case of s-wave pairing, for the lifetime the values $25,5.5$, and 1.8 , for $\mathbf{P}=0$ and $T / T_{c}$ equal to $1.5,3$ and 6 , respectively. This lifetime is expressed in units of the inverse of the Fermi energy $E_{F}$, where $E_{F}=\mu-E_{\text {bottom }}$ and $E_{\text {bottom }}$ is the bottom of the band. Although the pair structure function is defined on a lattice, we present it as a continuous function, as a guideline for the eye to follow its dependence on the relative distance. We will study separately the behaviour of $g_{P}(\rho)$ for small and for large values of $\rho$. First we will present figures, obtained by treating numerically the relevant equations presented in Section 2, which show the influence of various parameters, such as center of mass momentum, chemical potential, attraction strength, on the form of $g_{P}(\rho)$ up to a few lattice constants. The asymptotic behaviour for large distances, on the other hand, will be discussed by analyzing the basic integral over wave vectors in the bubble (Eq. (20)) that determines the $\rho$ dependence of $g_{P}(\rho)$.

We first present results for an isotropic interaction of the form (10) leading to s-wave pairing. It turns out that similar findings will apply correspondingly to the d-wave case, for which we will present the basic features in the following subsection. The last subsection will be devoted to the preformed pairs below $T_{c}$.

\section{1 s-wave pairing above $T_{c}$}

In Figure 1, we present the pair structure function up to three lattice constants for different values of the center of mass momentum in absence and in presence of a pseudogap. The function is calculated for the relative distance in the same direction as the momentum. In the s-wave case, however, the pair structure function is isotropic, reflecting the spatial symmetry of the order parameter, so that $g_{P}\left(\mathbf{P}, \rho_{x}\right)$ is representative of the behavior along the other directions. The effect of increasing pair momentum is to reduce the number of pairs and to shrink their size (which can be seen as a sort of "relativistic length contraction"). It is apparent that the extension of the pairs in all the cases considered here is of the order of one or two lattice constants. In order to see the effect of the finite pseudogap on $g_{P}(\rho)$ for this range of distances we show $g_{P}\left(\mathbf{P}, \rho_{x}\right)$ in the two figures for a fixed ratio $T / T_{c}$, the transition temperature $T_{c}$ being calculated in each case using the Thouless criterion [15]. A finite pseudogap increases the number of pairs and slightly enlarges their size for zero internal momentum. For finite value of the momentum $\mathbf{P}$, though, the opening of a pseudogap seems to suppress the pairing. Had we taken the same temperature for both figures, rather than the same ratio $T / T_{c}$, we would find fewer pairs with pseudogap: for a given strength of thermal fluctuations (same $T$ ) it is more difficult to form pairs in the presence of a pseudogap, since the density of states near the chemical potential is decreased. Moreover, when we increase the temperature, keeping all the other parameters the same, the values of $g_{P}(\rho)$ simply decrease by keeping the same form of the $\rho$-dependence.

In Figure 2 we show $g_{P}(\rho)$ for different values of the chemical potential $\mu$. A smaller value has been chosen for the pseudogap in order to give more amplitude to the oscillations, which according to equation (45) — are damped by the presence of a pseudogap. As the chemical potential 


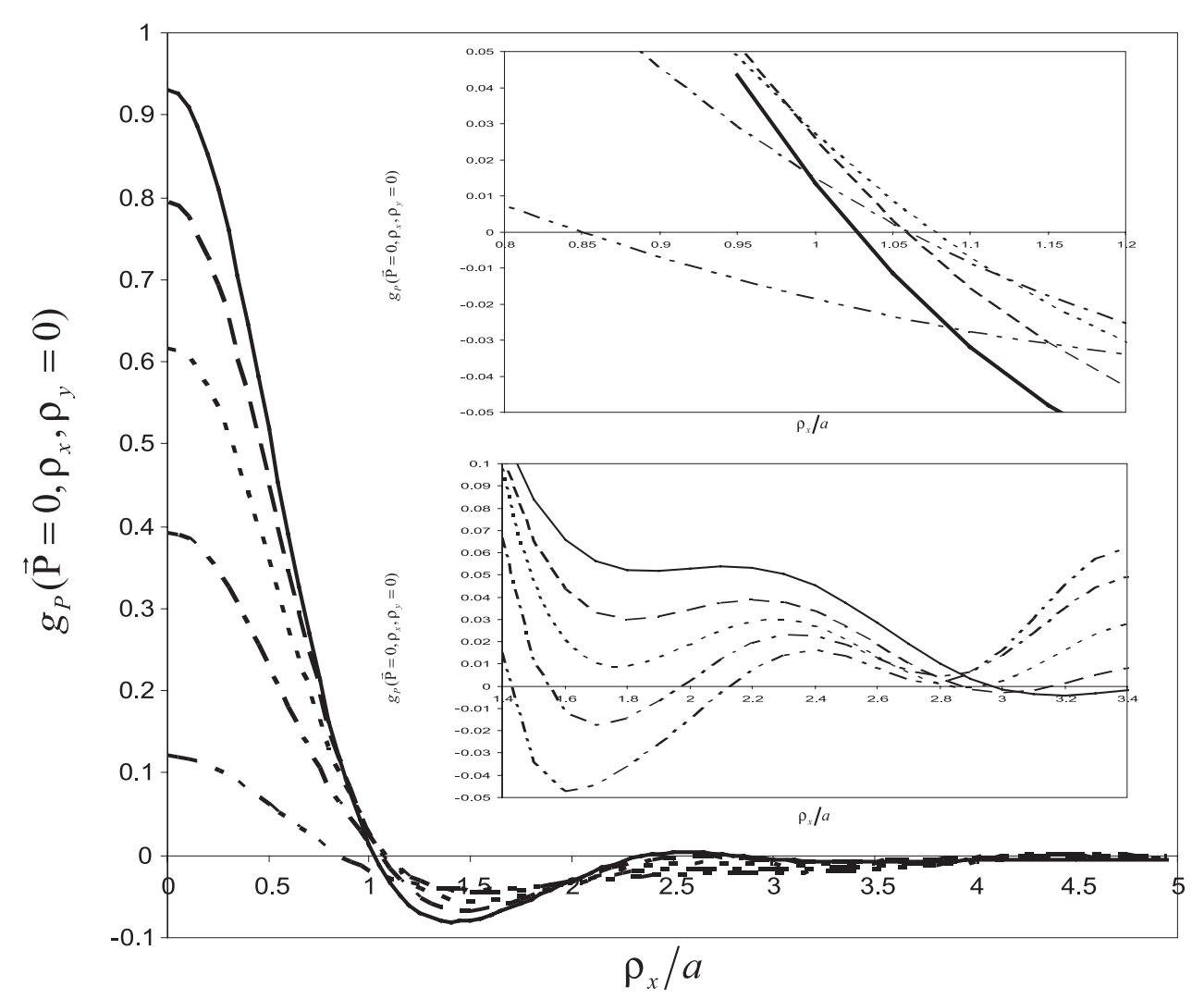

Fig. 2. Density dependence of the pair structure function $g_{P}(\mathbf{P}, \boldsymbol{\rho})$ for s-wave case, $\boldsymbol{\rho}=\left(\rho_{x}, 0\right), T=1.5 T_{c}, U=-0.5 W$, $k_{0} a=20$ and $\Delta_{p g}=0.0174 W$. Full line corresponds to $\mu=-0.35 W$, dashed line to $\mu=-0.375 W$, dotted line to $\mu=-0.4 W$, dash-dotted line to $\mu=-0.425 \mathrm{~W}$ and double dash-dotted line to $\mu=-0.45 \mathrm{~W}$.

moves up from the bottom of the band (i.e. for increasing density, but still in the limit of validity of our T-matrix approximation), the number of pairs with small $\rho_{x}$ also increases: there are more electrons available to form these pairs. Moreover, the fluctuating pairs get somewhat more localized: the curve for $g_{P}(\rho)$ reaches zero for a smaller $\rho_{x}$ and the following oscillations are correspondingly shifted. These oscillations are a signature of the Fermi sea out of which pairing takes place, just like the Friedel oscillations of a screened interaction potential.

In Figure 3 we show the variation of the pair structure with respect to variation of the interaction strength. We have chosen $\Delta=0$, since we are particularly interested in the behaviour of $g_{P}(\rho)$, when the interaction potential gets rather weak. When $U$ is decreased $g_{P}(\rho)$ loses amplitude: fewer fluctuating pairs are formed. However, the range of the main peak for small distances essentially remains unchanged when $U / W$ varies between -0.2 and -0.1 . The inset shows the pair structure functions for stronger potential strengths (between -0.2 and -0.6 ). The functions are normalized to their zero internal distance value, as we want to emphasize the trend of the functions. As one would expect, the typical internal distance increases as the interaction strength $U$ has weakened from $0.6 \mathrm{~W}$ to $0.2 \mathrm{~W}$.

This evolution is similar to what has been obtained by Paredes and Cirac [20] for a system of bosonic atoms in an optical lattice, which is mapped onto a fermionic

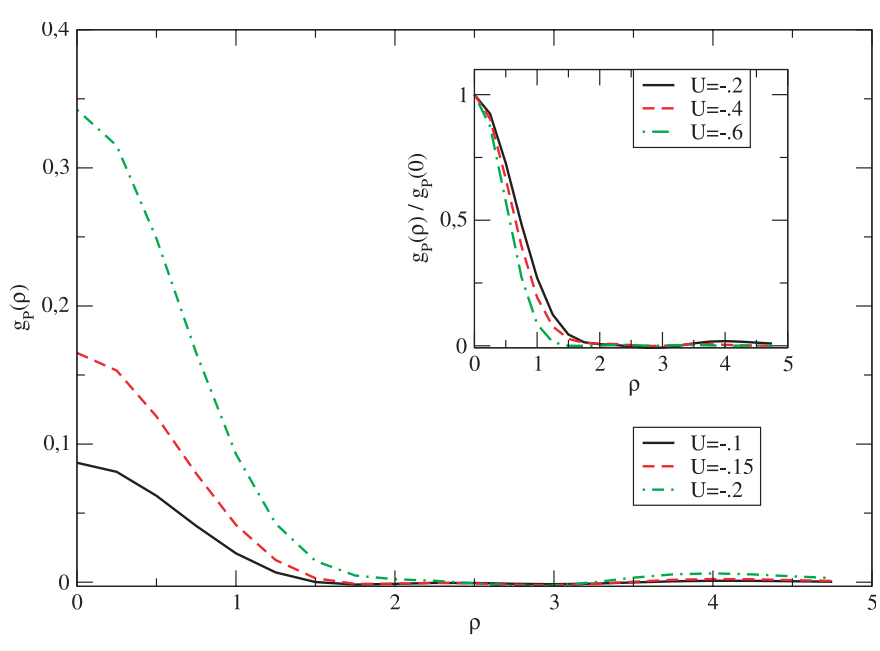

Fig. 3. Pair structure function $g_{P}(\mathbf{P}=\mathbf{0}, \boldsymbol{\rho})$ at $T=1.5 T_{c}$, for different interaction strengths: $U=-0.1 W,-0.15 W$, and $-0.2 W$. We have chosen $\Delta_{p g}=0$. The inset shows $g_{P}(\rho)$ for somewhat stronger attraction $(U=-0.2 W,-0.4 W$, and $-0.6 W)$. Here the structure function is normalized to one at the origin in order to show more clearly the variation of the short range form for different interaction strength.

system via a Jordan-Wigner transformation with a local attraction $V$. The pairs are formed according to a variational wave function, whose spatial extension changes by 
changing the attraction strength, and results in localized pairs for large value of $V$, and delocalized pairs for small $V$.

We have also studied the influence of the potential range on $g_{P}(\rho)$ by varying the parameter $k_{0}$ in the form function $\phi(\boldsymbol{k})$ of our factorized potential (9). One has to bear in mind, however, that, for smaller and smaller $k_{0}$, such a factorizable potential deviates more and more from a true distance dependent interaction, which would lead to a BS integral equation (7). The result is that for decreasing $k_{0}$, the amplitude of $g_{p}$ for small $\rho$ decreases and the oscillations taking place for $\rho$ up to a distance on the order of the inverse of $k_{0}$ disappear. However, the oscillations for larger distances get enhanced, such that for a potential with a longer range pairs tend to be less localized, as expected.

We now examine the $\rho$-dependence of $g_{P}(\rho)$ in more detail, looking also into the asymptotic behaviour for large distances. According to expression (14) the $\rho$-dependence comes from the "two-particle bubble" $\mathcal{G}$, given by the sum over wave vectors in equation (20).

First, we consider a weak attraction, where the pseudogap can be neglected. In this regime the zero momentum bubble has the form

$$
\mathcal{G}(\mathbf{0}, \boldsymbol{\rho}, z)=\int d^{2} k\left\{e^{i \boldsymbol{k} \cdot \boldsymbol{\rho}} \frac{\tanh \left[\frac{\beta(\epsilon(\boldsymbol{k})-\mu)}{2}\right] \phi(\boldsymbol{k})}{z-2(\epsilon(\boldsymbol{k})-\mu)}\right\}
$$

When the free electron spectrum and the form function $\phi(k)$ are approximated by an isotropic form, the imaginary part of the bubble for $z$ going to $\Omega+i \epsilon$ has the form:

$$
\operatorname{Im}[\mathcal{G}(\mathbf{0}, \boldsymbol{\rho}, \Omega+i \delta)]=2 \pi m J_{0}\left(k_{c} \rho\right) \tanh \left[k \frac{\beta \Omega}{4}\right] \phi\left(k_{c}\right)
$$

where $J_{0}(x)$ is the zero order Bessel function and the characteristic wave number $k_{c}$, given by the zero of the denominator in (38)

$$
\begin{aligned}
k_{c} & =\sqrt{2 m\left(\mu+\frac{\Omega}{2}\right)} \\
& =k_{F}(1+\Omega /(2 \mu))^{1 / 2}
\end{aligned}
$$

is close to the Fermi wave number $k_{F}$, since $\Omega$, corresponding to the pair energy over which we integrate in relation (21), is on the order of $k_{B} T$ and is therefore small. According to (14) the relevant quantity $\chi$, determining $g_{P}(\rho)$, involves the product of two such (complex valued) bubbles and since $k_{c}$ depends on the energy $\Omega$ over which we integrate in (21), the final asymptotic $\rho$-dependence of $g_{P}(\rho)$ cannot be immediately guessed from these considerations. Nevertheless, $k_{c}$ is a key quantity in order to understand the general shape of $g_{P}(\rho)$. First, it determines through the Bessel function form and width of the main peak of $g_{P}(\rho)$, and thus the characteristic length $d_{f p}$ which represents the typical size of preformed or fluctuating pairs, as it appears in our figures, is of the order of the inverse of $k_{c}$.
Our expression (14) for the pair structure function shows that, in the weak coupling limit, the influence of the interaction strength only shows up in the T-matrix expression (15) — but not explicitly in the two-particle bubble which alone depends on $\rho$. Thus the $\rho$-dependence of the pair structure function $g_{P}(\rho)$ will be governed by the characteristic length, given by $(40)$, whereas the potential strength only influences the amplitude of $g_{P}(\rho)$ through the T-matrix factor in (14).

This explains the relatively small variation of the pair size with a strongly varying $U$, shown in Figure 3 .

In order to compare the $\rho$ dependence for different values of $U$ the pair structure functions shown in the inset are scaled to one at the origin. For the chemical potential used ( $\mu$ is $0.8 t$ above the bottom of the band) the $k_{c}$ value given by equation (40) is on the order of the inverse of the lattice constant. This is indeed reflected in the $\rho$ dependence of $g_{P}(\rho)$, which varies little from one curve to another. On the other hand, the pair number strongly decreases then the attraction becomes weaker, as it is manifest looking at the values of $g_{P}(0)$ for curves at different interaction strengths.

The conclusion is that pairs, created out of the Fermi sea, remain relatively local even in the weak coupling limit. Their size is related to the inverse of the Fermi momentum $k_{F}$, since the dominant wave vectors of the two partners forming these pairs are near $k_{F}$. This is different from the case of an isolated pair, treated as a two-particle scattering problem. This corresponds to choosing $\mu=0$. In this case the relevant length scale as far as the pair size is concerned is the potential range; when the potential becomes weaker, the amplitude of the wave function gets smaller inside the attractive region and thus the pair state looks more extended.

This conclusion can also be reached by transforming the Bethe-Salpeter equation for the two-particle correlation function from its integral form (as it is shown in our Eqs. (7) and (12)) into a Schroedinger-like equation which, for an interaction potential $U(\boldsymbol{\rho})$, reads:

$$
\begin{aligned}
\left(z+\frac{\nabla_{\rho}^{2}}{m}\right) \chi\left(\mathbf{0}, \boldsymbol{\rho}, \boldsymbol{\rho}^{\prime}, \mathbf{z}\right) & =-i R\left(\boldsymbol{\rho}-\boldsymbol{\rho}^{\prime}\right) \\
& +\int d^{2} r R(\boldsymbol{\rho}-\mathbf{r}) \mathbf{U}(\mathbf{r}) \chi\left(\mathbf{0}, \mathbf{r}, \boldsymbol{\rho}^{\prime}, \mathbf{z}\right)
\end{aligned}
$$

where the function $R(\boldsymbol{\rho})$ is given by

$$
R(\boldsymbol{\rho})=\frac{1}{(2 \pi)^{2}} \int d^{2} k e^{-i \mathbf{k} \boldsymbol{\rho}}\left[1-2 N_{F}\left(k^{2} / 2 m\right)\right] .
$$

In the dilute limit the Fermi functions do not contribute to $R(\boldsymbol{\rho})$, which leads to $R(\boldsymbol{\rho})=\delta(\boldsymbol{\rho})$. Equation (41) then takes the usual Lipmann-Schwinger form describing twoparticle scattering (see Chap. 13 of Ref. [15]). However, for a Fermi system of finite $\mu$ our scattering equation (41) remains an integro-differential equation and, for a short range potential, the integral kernel is again dominated by the Fermi wave vector determining the range of $R(\rho)$. 
The asymptotic behaviour of $g_{P}(\rho)$ in the weak coupling limit can also be discussed with the help of $k_{c}$. For large distances $I m \mathcal{G}$ has oscillations of the form $\frac{\cos k_{c} \rho}{\rho}$. For $g_{P}(\rho)$ we indeed find numerically, for large $\rho$, oscillations with wavelength on the order of a few lattice constants. Their amplitude decreases as $\rho^{-s}$ with an exponent $s$ which is somewhat larger than one. Its precise value depends on the chosen parameters, in particular on temperature. This shows that the second moment in $\rho$ of $g_{P}(\rho)$ does not exist, and thus no "mean fluctuating pair size" can be defined in the weak coupling limit. However, since $s>1$ the integral of $g_{P}(\rho)$ over $\rho$ exists and can in principle be interpreted as the total weight of all pair configurations for a given center of mass momentum $\mathbf{P}$. With increasing temperature the amplitude of these oscillations decreases, their form changing only slightly. The asymptotic behaviour of $g_{P}(\mathbf{P}, \boldsymbol{\rho})$ is not surprising. Indeed, the Bethe-Salpeter equation we use for calculating the two-particle Green function describes the scattering of two electrons, due to their attractive interaction, in the presence of the Fermi sea. Our $g_{P}(\rho)$ gives then the difference in the number of paired configurations, with the partners being at a distance $\rho$, between the interacting system and the background of non-interacting paricles. The corresponding difference between the squares of the wave function describing a scattering state and of the free state of the same energy (in the absence of a Fermi sea) decreases asymptotically as $\rho^{-1}$ in two dimensions.

Thus, for weak coupling the fluctuating pairs look different from the pairs forming the superconducting condensate [21], where a characteristic mean pair length $\xi_{\text {pair }}$ can be defined by the normalized second moment of the probability distribution $g_{a}(\rho)$ for the distance $\rho$ between the partners of the pair. In the BCS approach the latter is given by the Fourier transform to real space of the equal-time anomalous Green function [21]. It is given by an integral like our expression (38), but for $z=0$ and $\xi(\boldsymbol{k})$ replaced by the gapped electron spectrum. For a finite superconducting gap $\Delta_{s c}$ the function $g_{a}(\rho)$ decreases exponentially for large distances and the characteristic pair size $\xi_{\text {pair }}$ found in this way [21] is given by

$$
\xi_{\text {pair }}=\hbar^{2} \frac{k_{F}}{4 m \Delta_{s c}} .
$$

Except for a slightly different numerical factor in the denominator this is the usual BCS coherence length. Note that equation (2.69) of reference [21] has a slightly different form, since these authors use a scaled gap.

We now consider an attraction $U$ of intermediate value (on the order of half the bandwidth or more). It will produce a pseudogap in the one-electron Green function. The corresponding bubble, replacing (38), is shown in appendix A in equation (53). Due to the BCS-form (50) of $G$ four terms contribute to the bubble in (53). The first two are of particle-particle type like the bubble (38) for free electrons. The other two are particle-hole like, but since they involve the product of the two BCS, respectively Bogoliubov weight functions $u_{\mathbf{k}}$ and $v_{\mathbf{k}}$, they contribute less. For the particle-particle terms the relevant wave number $k_{c}$, for which the corresponding denominator in the bubble (53) vanishes, will now be complex involving the value $\Delta_{p g}$ of the pseudogap:

$$
k_{c}=k_{F}+i m\left(\Delta_{p g}^{2}+\Gamma^{2}\right)^{1 / 2} / \hbar k_{F} .
$$

In this expression we have neglected the energy $\Omega$ of the pairs. For a pseudogap which is somewhat smaller than the chemical potential (as it seems to be the case in underdoped cuprates) the real part of $k_{c}$ is still dominant, such that the short range structure of preformed pairs will again be characterized by a length $d_{f p}$ on the order of the inverse Fermi wave number. However, the imaginary part of $k_{c}$ now leads, through the properties of the Bessel function of a complex argument, to an exponential decay of $g(\rho)$ for large $\rho$. The same exponential decay would, of course, be found for the spatial Fourier transform of the one-electron spectral function (49) for energies $\Omega$ smaller than the value of the pseudogap.

We can now introduce a fluctuating pair length $\xi_{f p}$ which differs from the quantity $d_{f p}$. It is given by

$$
\xi_{f p}=\hbar^{2} \frac{k_{F}}{4 m \sqrt{\Delta_{p g}^{2}+\Gamma^{2}}} .
$$

On the other hand the structure of condensed pairs in this regime is similar as in the weak coupling limit: the electronic band structure governing the anomalous Green functions describing the superconducting phase is now characterized by a total gap which is composed of the pseudogap and the superconducting gap. A simple form used by various authors is

$$
\Delta^{2}=\Delta_{s c}^{2}+\Delta_{p g}^{2}
$$

and expression (41) has to be replaced by

$$
\xi_{\text {pair }}=\hbar^{2} \frac{k_{F}}{4 m \Delta} .
$$

Thus, the condensed pairs now have a correlation length which is not very different from the one of the preformed pairs existing above the transition and in this regime the idea that fluctuating pairs condense when superconductivity sets in begins to be relevant. This has also been shown by thermodynamic arguments [16].

What is usually called strong coupling [21] corresponds to an attraction $U^{*}$ exceeding the band width, which leads to a chemical potential lying far below the lower band edge. One can then show [21] that the two-electron propagator takes the form of the Green function of a Boson corresponding to strongly localized electron pairs. On the other hand the one-electron spectrum in the bubble can be neglected with respect to $\mu$ and the $k$-integral over the Bessel function yields again strongly localized preformed pairs, $\xi_{f p}$ now being of the order of the inverse of a wave vector on the edge of the Brillouin zone. Thus, for strong coupling, condensed pairs are as localized as the fluctuating ones, which legitimates the Bose-Einsteincondensation scenario for the superconducting transition in this regime. 
(a)

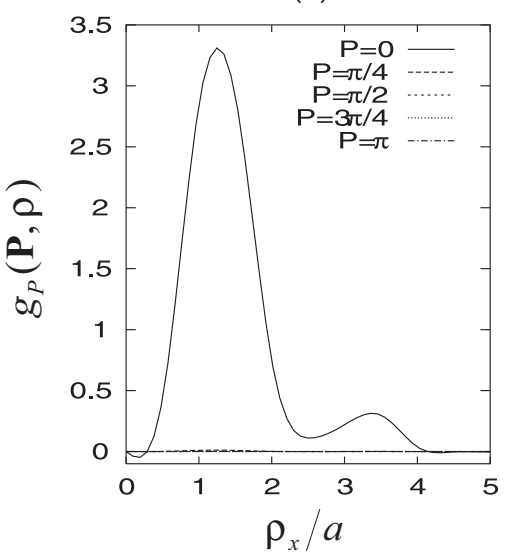

(b)

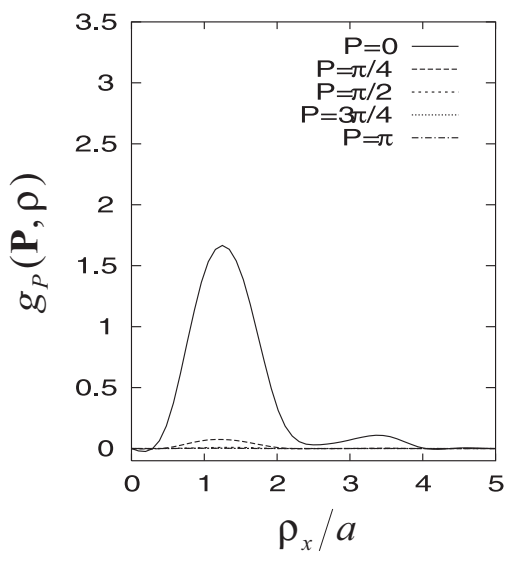

Fig. 4. Temperature dependence of the pair structure function $g_{P}(\mathbf{P}, \boldsymbol{\rho})$ for the $d$-wave symmetry as a function of $\rho_{x}$ and different values of the center of mass momentum $\mathbf{P}$. The following parameters have been used: $\Delta_{p g}=0.05 \mathrm{~W}$, $\mu=-0.4 W, U=-0.7 \mathrm{~W}$ and the temperature values are $T=1.5 T_{c}$ in (a) and $T=3 T_{c}$ in (b).

\section{$3.2 \mathrm{~d}$-wave pairing above $\mathrm{T}_{\mathrm{c}}$}

As stated above, the d-wave case does not differ too much in the qualitative behavior from the previous cases, neither in the behavior with respect to the opening of a pseudogap in the single particle spectrum, nor in the dependence on the other parameters. We just show in Figure 4 the variation with temperature dependence of $g_{P}(\rho)$ for different momenta. As the temperature is raised, the distribution function is suppressed for $\mathbf{P}=0$ and enhanced for $P \neq 0$, as in the s-wave case. $g_{P}(\rho)$ vanishes for zero internal distance, as it should be from the symmetry of the interaction and of the pseudogap parameter, and has its maximum at a distance of the order of a lattice constant, slightly higher than the extended s-wave case. The d-wave symmetry is also visible in the fact that $g_{P}(\rho)$ is zero along the diagonal $\left(\rho_{x}=\rho_{y}\right)$, see Figure 5 . The pairs again have typically the size of a lattice constant, but a secondary peak appears in the zero momentum distribution function at a distance of three or four lattice constants. Another fact to be noticed is that when a gap opens we need to move toward stronger interaction value in order to have a reasonable value for the transition temperature. This is due to the fact that a finite value of the interaction in the 2D Hubbard model is needed for the d-wave case to have a bound state in the related two-body problem [18]. Moreover, the Friedel oscillations, discussed above for the s-wave case, are hidden by the oscillation of the d-wave symmetry of pseudogap parameter, of typical period $\pi / a$.

By lowering the interaction strength the number of pairs tends to diminish strongly. Moving the chemical potential more inside the band has as a result an enhancement of the number of pairs of finite momentum. To explain this enhancement, we can invoke the increase of kinetic energy due to confinement. We can also note a decrease of the average extension of the pair, as the secondary peak in the pair structure function almost completely disappears.

The asymptotic behaviour of $g_{P}(\rho)$ for large $\rho$ is also similar as for s-wave pairing: in the absence of a pseudogap it goes to zero as a power of $\rho$, whereas the presence of a pseudogap again leads to an exponential decay characterized by the corresponding $\xi_{f p}$.

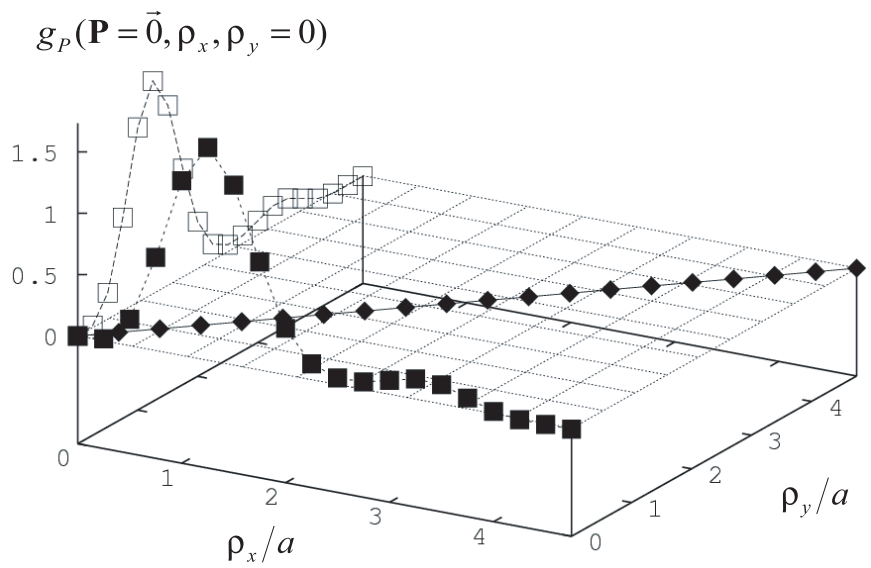

Fig. 5. Spatial two-dimensional structure of the pair structure function $g_{P}(\mathbf{P}=\mathbf{0}, \boldsymbol{\rho})$ for different directions of $\boldsymbol{\rho}$ for the dwave symmetry case. The parameter values are: $\Delta_{p g}=0.05 \mathrm{~W}$, $T=1.5 T_{c}, \mu=-0.4 W$ and $U=-0.7 W$.

\subsection{Below the transition temperature}

In Figure 6 we compare the distribution functions $g_{a}$ of pairs in the condensate, given by the "anomalous" bubble $G\left(x_{2} ; x_{1}\right)_{12} G\left(x_{1}^{\prime} ; x_{2}^{\prime}\right)_{21}$ in expression (32) and discussed in Section 2, for s- and d-wave symmetries. It is from this function that a pair length [21,10], which represents the average distance among fermions in a Cooper pair (see Appendix C), is usually defined.

The structure of fluctuating pairs below $T_{c}$, given by the second contribution to (28), are presented in Figure 7 for the s- and d-wave symmetry of the order parameter for temperatures around $T_{c}$. We can thus assume that $\Delta(\mathbf{k})$ remains almost constant, with a value corresponding to the one in the pseudogap state [23], while the value of the superconducting order parameter $\Delta_{s c}(\mathbf{k})$ builds up from zero at the transition temperature and increases to its zero temperature value following a $1-\left(T / T_{c}\right)^{1 / 2}$ increasing law.

One can see that the number of fluctuating Cooper pairs is suppressed as the long range order develops, i.e. it decreases with the temperature, contrary to the behavior above $T_{c}$ and consistently to what expected. The spatial 
(a)

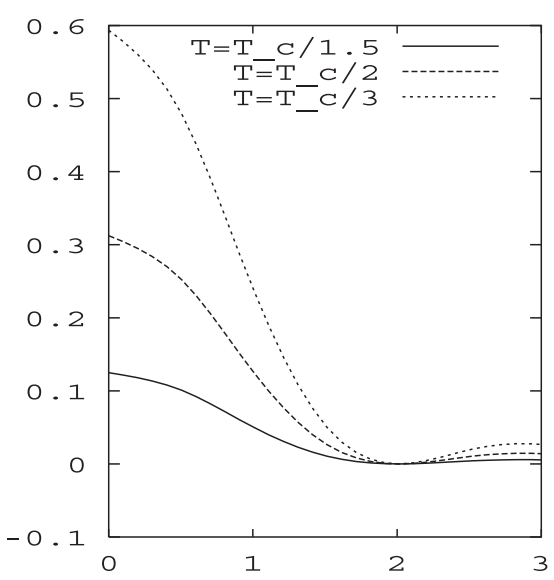

(b)

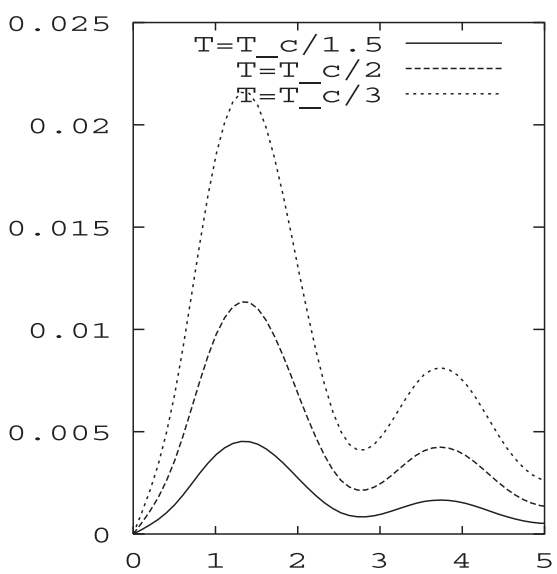

Fig. 6. Pair structure function $g_{a}(\boldsymbol{\rho})$, for pairs in the superconducting condensate, as a function of temperature for: (a) s-wave symmetry and (b) d-wave symmetry. The parameters for the gap, the chemical potential and the interaction strength are as in Figures 3a and 4a for s- and d-wave respectively. The total pair number increases when the temperature is lowered, as expected.

(a)

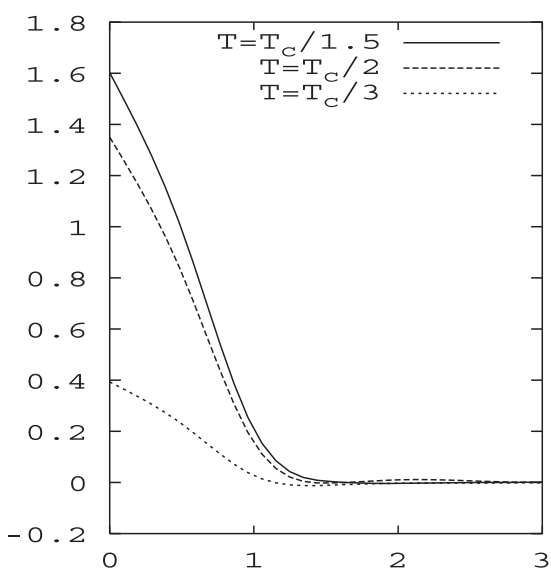

(b)

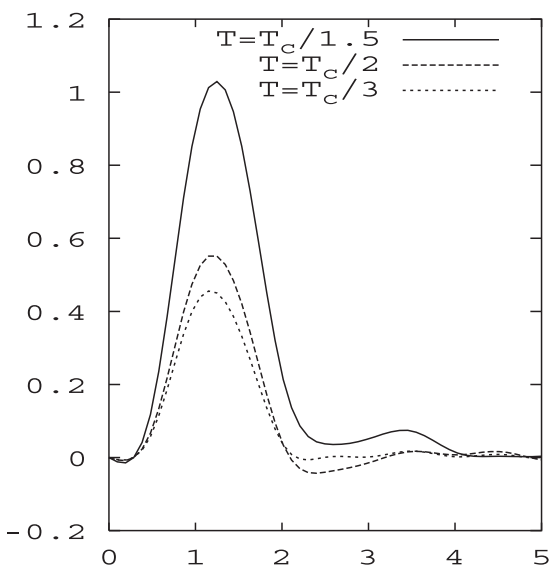

Fig. 7. Pair structure function $g_{P}(\mathbf{P}=\mathbf{0}, \boldsymbol{\rho})$, for fluctuating pairs in the superconducting state, as a function of temperature for: (a) s-wave symmetry; (b) d-wave symmetry. The parameters for the gap, the chemical potential and the interaction strength are as in Figures $3 \mathrm{a}$ and $4 \mathrm{a}$ for s- and d-wave respectively. The total number of fluctuating pairs decreases when the temperature is lowered, which is the opposite behaviour of what is shown in Figure 6.

extension of the pair structure function does not change appreciably, and it is somewhat shorter than the one inferable from the inspection of the distribution function $g_{a}(\rho)$ in Figure 6. However, it should be pointed out that a comparison between the two functions can not be but qualitative, since $g_{a}(\rho)$ is a probability distribution function (though not yet normalized to one), while $g_{P}(\mathbf{P}, \boldsymbol{\rho})$ is not a positively-defined function.

\section{Discussion}

In this work, we have proposed a function that describes the internal structure of fluctuating pairs in the weak coupling and in the pseudogap regions of high temperature superconductors and in the broken symmetry phase in the vicinity of the transition temperature. We assume that the system can be approximately described by an attractive Hubbard model. In this framework, we have studied the Green function for the operator which creates a pair of particles whose partners are at a certain relative distance. In order to describe fluctuating pairs we applied the Bethe-Salpeter approach and the corresponding $T$-matrix formalism, which takes into account the relevant interaction channel of repeated two-particle scattering in the presence of the Fermi sea.

We describe the pairs in terms of three variables: energy $\Omega$, center of mass momentum $\mathbf{P}$ and internal distance $\boldsymbol{\rho}$ between the partners. Integrating over $\Omega$ with a Bose distribution we obtain a pair structure function $g_{P}(\mathbf{P}, \boldsymbol{\rho})$ giving information about the number of pairs having momentum $\mathbf{P}$ and internal distance $\boldsymbol{\rho}$. The expression that determines $g_{P}(\rho)$ has two ingredients: the $T$-matrix brings in the spectral properties of pairs with a fixed value of $\boldsymbol{\rho}$, whereas the $\boldsymbol{\rho}$ dependence we are interested in here is brought in by the two-particle bubble. Strictly speaking, $g_{P}(\rho)$ is not a probability distribution, since we subtract the uniform background for non-interacting particles. Positive (negative) values of $g_{P}(\mathbf{P}, \boldsymbol{\rho})$ then indicate 
an enhanced (reduced) tendency of the system of forming pair configurations in the presence of the attraction. We have studied the behaviour of $g_{P}(\mathbf{P}, \boldsymbol{\rho})$ when its arguments (range $\rho$ and momentum $\mathbf{P}$ ), as well as various parameters, namely temperature $T$, interaction strength $U$ and chemical potential $\mu$ (and thus electronic density), are modified. For the pseudogap in the one electron spectral functions we have used values which have been obtained by quantum Monte Carlo simulations of the Hubbard model [13].

Here are our results grouped according to the dependence of the pair structure function $g_{P}(\rho)$ on different parameters:

a) Distance $\rho$ between the two partners

When $g_{P}(\rho)$ is plotted as a function of $\rho$, as it is shown in our figures, most pairs seem to be relatively localized, extending only over one or two lattice constants. However, in order to appreciate the number of pairs of different size, the asymptotic behaviour of the function $g_{P}(\rho)$ for the fluctuating pairs has to be examined. It depends on the interaction strength $U$ :

For small $U$ (weak coupling) $g_{P}(\rho)$ has a long tail in $\rho$ with Friedel-like oscillations, the amplitude of which decays as $\rho^{-s}$ with an exponent s lying between 1 and 2 , depending on the parameter values chosen. It is thus not possible to use the second moment of $\rho$ in order to define a mean pair size. Moreover, when the total number of fluctuating pairs is calculated as an integral over $\rho$ large pairs will give an important contribution due to the non-exponential decay of $g_{P}(\rho)$.

When $U$ is larger (intermediate and strong coupling), leading to a pseudogap in the one-electron spectral function, $g_{P}(\rho)$ goes to zero exponentially for large $\rho$. The second moment of $g_{P}(\rho)$ corresponds to a fluctuating pair correlation length $\xi_{f p}$ given by expression (46). It becomes shorter when the pseudogap increases and the fraction of relatively localized pairs becomes more important.

The symmetry of the pairing interaction is reflected in the main structure of $g_{P}(\rho)$ as a function of $\rho$ : for s-wave pairs the maximum is at $\rho=0$, whereas for d-wave pairs $g_{P}(\rho)$ goes to zero for $\rho$ going to zero. Moreover, the dependence on the direction of $\rho$ corresponds to d-wave symmetry.

b) Center of mass momentum $\mathbf{P}$

$g_{P}(\mathbf{P}, \rho)$ decreases motonically when $\mathbf{P}$ increases: most pairs have zero momentum. Pairs with finite momentum are thus typically somewhat smaller than pairs at rest.

c) Temperature $T$

Above the superconducting transition temperature $T_{c}$ increasing $T$, and thus the strength of thermal fluctuations, reduces the value of $g_{P}(\mathbf{P}, \rho)$ : the number of fluctuating pairs decreases. Below $T_{c}$ there are Cooper pairs with a finite mean size forming the superconducting condensate. However, there are still fluctuating pairs below $T_{c}$. They show the opposite behaviour temperature dependence: reducing $\mathrm{T}$ leads to fewer fluctuating pairs, whereas the number of condensed pairs increases. d) Strength and form of the interaction potential

For a fixed ratio $T / T_{c}$ a weaker interaction $U$ leads to a lower number of pairs. Moreover, the pairs get somewhat less localized when the attraction is weaker. However, it is important to stress that the characteristic length, given in the weak coupling limit by equation (40), determining the short range form of $\rho$ mainly depends on the electron density, rather than on $U$. Thus, the structure of pairs formed on top of the Fermi sea is different from what one expects in the framework of scattering theory that describes the resonance states of two isolated particles.

Going from a local attraction to an interaction of longer range in the framework of a separable potential is achieved by making the characteristic wave number $k_{0}$ in expression (10) smaller. Pairs then get wider and the long range Friedel oscillations are enhanced.

e) Chemical potential

When $\mu$ moves down towards the lower band edge the number of pairs is reduced, there being fewer electrons around to form them. Moreover, the long range oscillations in $\rho$, observed for weak coupling, change in amplitude and wave length (as it is the case for the Friedel oscillations of the screened interaction). When the electronic density goes to zero the $U$-dependence expected from scattering theory is recovered.

In the framework of the attractive Hubbard model preformed, or fluctuating pairs have widely been treated by analyzing, for example, the properties of the T-matrix or by transforming the partition function into a functional integral over a pairing field. However, as stated above, the usual T-matrix, as well as the pairing field, only depends on energy and center of mass momentum of the pairs. One thus only describes pairs with fixed, characteristic internal distance $\rho$ between the partners. For the usual on-site attraction the pairs treated like this are local, the partners sitting on the same lattice site. Such an approach thus does not give information about the internal structure of the fluctuating pairs, as it is given by our pair structure function.

It would, of course, be interesting to observe the distribution of internal distances for the pairs of high temperature superconductors. An indirect access to the pair correlation function, which is equivalent to our pair structure function integrated over all the centre of mass momenta, is possible by the fact that in the broken symmetry phase it is connected to the correlation energy [24]. This energy, in turn, plays a key role in the thermodynamics of the phase transition itself. The real space picture of this pair correlation function, reported in [24] looks similar to what we obtained for $g_{P}(\mathbf{P}, \boldsymbol{\rho})$ both for s- and d-wave case as can be seen by comparing with Figure 7 (we point out that in contrast to reference [24], we reported the values of the pair structure function not only on the lattice sites, but also on intermediate positions).

Another area for which the internal structure of Cooper pairs may be relevant is the very active field of systems of ultracold atoms. In fact, while it is probably difficult to measure the internal structure of the Cooper 
pairs in a superconductor, due to the very complicated structure of the cuprates, in the case of atoms trapped in optical lattice it is possible to carefully control all the parameters for the interaction among particles [19]. Moreover, it should be possible to actually measure the extension of the Cooper pairs. Altman et al. [28] have explained how the analysis of the light absorption image of a cloud of such ultracold atoms, expanding when the trapping potential has been turned off, can be used in order to study the momentum distribution of the trapped atom system. They show that, in the framework of a BCS-like state such a measurement would yield information about the usual functions $u(k)$ and $v(k)$ used to build up the state. These functions determine the structure of the condensed pairs. In the same way one would obtain insight into our structure function $g_{P}(\rho)$ for fluctuating pairs. Alternatively, one could analyze the response to a Raman laser which couples to the internal states of the pair, as suggested in $[20]$.

We thank S. Sharapov, P. Pieri, G.C. Strinati and L. Benfatto for helpful discussions. This work was supported by the National Competence Centre "MaNEP" of the Swiss National Science Foundation.

\section{Appendix A: Spectral function in the pseudogap region}

To mimic the presence of a pseudogap, we consider the form of the BCS Green function with a finite linewidth. Due to the absence of long range order the "anomalous" BCS Green function is zero. We have then:

$$
G(\mathbf{k}, z)=\frac{1}{z-\xi(\mathbf{k})+i \Gamma-\frac{\Delta^{2}(\mathbf{k})}{z-\xi(\mathbf{k})+i \Gamma}}
$$

where $\xi(\mathbf{k})=-2 t\left(\cos k_{x}+\cos k_{y}\right)$ is the single particle dispersion, $1 / \Gamma$ is the excitation lifetime and $\Delta(\mathbf{k})$ is the pseudogap. It is possible to rewrite (48) in terms of the quasiparticles excitation energy, that is $E(\mathbf{k})=$ $\sqrt{\xi^{2}\left(\mathbf{k}+\Delta^{2}(\mathbf{k})\right.}$ :

$$
\begin{aligned}
G(\mathbf{k}, z) & =\frac{z+\xi(\mathbf{k})+i \Gamma}{(z+E(\mathbf{k})+i \Gamma)(z-E(\mathbf{k})+i \Gamma)} \\
& =\frac{u_{\mathbf{k}}^{2}}{z-E(\mathbf{k})+i \Gamma}+\frac{v_{\mathbf{k}}^{2}}{z+E(\mathbf{k})+i \Gamma}
\end{aligned}
$$

where $u_{\mathbf{k}}^{2}=(E(\mathbf{k})+\xi(\mathbf{k})) /(2 E(\mathbf{k}))$ and $v_{\mathbf{k}}^{2}=(E(\mathbf{k})-$ $\xi(\mathbf{k})) /(2 E(\mathbf{k}))$. From (49) we can deduce the form of the spectral function:

$A(\mathbf{k}, \Omega)=\frac{1}{\pi}\left(\frac{u_{\mathbf{k}}^{2} \Gamma}{(\Omega-E(\mathbf{k}))^{2}+\Gamma^{2}}+\frac{v_{\mathbf{k}}^{2} \Gamma}{(\Omega+E(\mathbf{k}))^{2}+\Gamma^{2}}\right)$.
The particle-particle bubble can be written in term of the spectral function of the single particle Green functions:

$$
\begin{aligned}
\mathcal{G}(\mathbf{P}, \mathbf{k}, z)= & \sum_{z_{\nu}} G\left(\mathbf{k}+\frac{\mathbf{P}}{2}, z_{\nu}\right) G\left(\mathbf{k}-\frac{\mathbf{P}}{2}, z-z_{\nu}\right) \\
= & \int_{0}^{\infty} \frac{d \omega}{2 \pi} A\left(\mathbf{k}+\frac{\mathbf{P}}{2}, \omega\right) \int_{0}^{\infty} \frac{d \omega^{\prime}}{2 \pi} A\left(\mathbf{k}-\frac{\mathbf{P}}{2}, \omega^{\prime}\right) \\
& \times \frac{f_{F}(\omega)+f_{F}\left(\omega^{\prime}\right)-1}{z-\omega-\omega^{\prime}} .
\end{aligned}
$$

Replacing the expression for the single particle spectral function (50) into the previous equation and performing the substitution $z \rightarrow \Omega+i \delta$, we can obtain the expression for the spectral function of the particle-particle bubble $I(\mathbf{P}, \mathbf{k}, \Omega)$ :

$$
\begin{aligned}
& I(\mathbf{P}, \mathbf{k}, \Omega) \equiv \operatorname{Im} \mathcal{G}(\mathbf{P}, \mathbf{k}, \Omega+i \delta)= \\
& \frac{2}{\pi}\left(\frac{u_{1}^{2} u_{2}^{2} \Gamma}{\left(\Omega-\left(E_{1}+E_{2}\right)\right)^{2}+4 \Gamma^{2}}+\frac{v_{1}^{2} v_{2}^{2} \Gamma}{\left(\Omega+\left(E_{1}+E_{2}\right)\right)^{2}+4 \Gamma^{2}}\right. \\
& \left.+\frac{u_{1}^{2} v_{2}^{2} \Gamma}{\left(\Omega-\left(E_{1}-E_{2}\right)\right)^{2}+4 \Gamma^{2}}+\frac{v_{2}^{2} \Gamma}{\left(\Omega+\left(E_{1}-E_{2}\right)\right)^{2}+4 \Gamma^{2}}\right),
\end{aligned}
$$

where we used the shorthand notation $1=\mathbf{k}+\mathbf{P} / 2$ and $2=\mathbf{k}-\mathbf{P} / 2$. The parameter that enters our calculations and that mimics the pseudogap is then the linewidth $\gamma=$ $2 \Gamma$ for the excitation described by the particle-particle bubble.

\section{Appendix B: Number of preformed pairs}

In this appendix we establish a relationship between our pair correlation function (3) and what is usually referred to as $N_{C P}$, the number of fluctuating Cooper pairs. In the framework of the usual Landau-Ginzburg (LG) description of a superconductor $N_{C P}$ is found by summing over the number $N_{C P}(\mathbf{P})$ of pairs with center of mass momentum $\mathbf{P}$, which is obtained by averaging the fluctuations of the pairing field (see, for example [25]):

$$
\frac{N_{C P}(\mathbf{P})=\sum_{\boldsymbol{k}}\left\langle\left|\psi_{\boldsymbol{k}}^{(f l)}\right|^{2}\right\rangle=\int d\left|\psi_{\boldsymbol{k}}\right|^{2}\left[P(\boldsymbol{k})\left|\psi_{\boldsymbol{k}}\right|^{2}\right]}{\int d\left|\psi_{\boldsymbol{k}}\right|^{2}[P(\boldsymbol{k})]} .
$$

Here $\psi_{k}(f l)$ is the fluctuating part of the Fourier transform of the pairing field governed by the static LG free energy and the average is taken over a probability distribution $\rho_{p}(P)$ given in terms of the coefficients in (16)

$$
P(\boldsymbol{k}) \propto \exp \left[-\left(a+c \boldsymbol{k}^{2}\right)\left|\psi_{\boldsymbol{k}}\right|^{2}\right]
$$

which yields

$$
N_{C P}(\mathbf{P})=1 /\left(a+c \mathbf{P}^{2}\right) .
$$

The number of fluctuating Cooper pairs has been obtained measuring the Knight shift and the nuclear spin relaxation under magnetic field by Zheng and co-workers [27]. 
These quantities reflect indeed the pseudogap behavior, and their reduction above $T_{c}$ is strongly magnetic field dependent accordingly to a scaling relation that depends on the effects of the Cooper pair density fluctuations [27].

Starting from our Hamiltonian (1) with the factorizable form (9) for the attractive potential (with one single shape function) one can transform the partition function into a functional integral by means of a Stratonovich-Hubbard transformation:

$$
\begin{aligned}
Z= & \operatorname{Tr} e^{-\beta H}=\operatorname{Tr} e^{-\beta H_{0}} \mathcal{T} \\
& \times \int \mathcal{D}^{2} \psi \exp \left[-i \int_{0}^{-i \beta} \sum_{\mathbf{P}}\left|U_{\lambda}\right|^{-1}|\psi(\mathbf{P}, \tau)|^{2}\right. \\
& \left.+\left(Q_{0}^{\dagger}(\mathbf{P}, \tau) \psi(\mathbf{P}, \tau) Q_{0}(\mathbf{P}, \tau) \psi^{*}(\mathbf{P}, \tau)\right)\right] d \tau
\end{aligned}
$$

Here $U_{\lambda}$ is the potential strength in equation (9) and $\psi(\mathbf{P}, \tau)$ is the pairing field accompanying the creation and annihilation operators of pairs, related to the basic definition (2) by

$$
Q_{0}(\boldsymbol{P})=\sum_{\boldsymbol{r}_{l}} e^{i \frac{\boldsymbol{P}}{2} \cdot \boldsymbol{r}_{l}} \sum_{\boldsymbol{\rho}_{l}} \sum_{\boldsymbol{k}} \phi_{\lambda}(\boldsymbol{k}) e^{-i \boldsymbol{k} \cdot \boldsymbol{\rho}_{l}} Q^{\dagger}(\boldsymbol{r}, \boldsymbol{\rho}) .
$$

We stress that $Q_{0}^{\dagger}$ creates reference pairs with a particular, fixed internal structure obtained by summing over pairs with different distance $\rho$ between partners, weighted by the space Fourier transform $\psi_{\lambda}(\boldsymbol{\rho})$ of the form factor $\psi_{\lambda}(\boldsymbol{k})$ of the interaction potential (see Eq. (54)). The dynamic correlation function of these reference pairs can be found from equation (12) by performing the same summation over $\boldsymbol{\rho}$ and $\boldsymbol{\rho}^{\prime}$ as in (54):

$$
\begin{aligned}
\chi_{0}\left(\boldsymbol{P}, z_{\nu}\right) & =\sum_{\boldsymbol{\rho}_{l}, \boldsymbol{\rho}_{l}^{\prime}} \chi\left(\boldsymbol{P}, \boldsymbol{\rho}_{l}, \boldsymbol{\rho}_{l}^{\prime}, z_{\nu}\right) \tilde{\phi}_{\lambda}\left(\boldsymbol{\rho}_{l}\right) \tilde{\phi}_{\lambda}\left(\boldsymbol{\rho}_{l}^{\prime}\right) \\
& =\frac{1}{U_{\lambda}} \sum_{\boldsymbol{k}}\left|\phi_{\lambda}(\boldsymbol{k})\right|^{2} \mathcal{G}\left(\boldsymbol{P}, \boldsymbol{k}, z_{\nu}\right) T\left(\boldsymbol{P}, z_{\nu}\right) .
\end{aligned}
$$

The number $N_{0}(\mathbf{P})$ of such reference pairs is given by

$$
N_{0}(\mathbf{P})=\sum_{z_{\nu}} \chi_{0}\left(\mathbf{P}, z_{\nu}\right)
$$

This is not identical to (55). However, treating pairs as classical, as usually in the LG approach, one can limit the sum to $z=0$, and taking into account that the factor multiplying the T-matrix in (58) varies slowly over the region where $\mathrm{T}$ is important, the excplicit form of the latter given in (16) leads us back to (55).

It is important to stress again that $N_{c p}(\mathbf{P})$ is the total number of pairs with a fixed internal structure, adapted to the form of the interaction in the starting Hamiltonian. It is thus not equal to what would be, in our approach, the total number of pairs with a given center of mass momentum, summed over all possible internal distance between the partners. This quantity would have to be identified with the integral over $\boldsymbol{\rho}$ of our pair distribution function $g_{P}(\mathbf{P}, \boldsymbol{\rho})$. The latter derives from a correlation function $\chi$ of the form (12) which is not identical with expression (58).

\section{Appendix C: Cooper-pair size at finite temperature}

In this appendix we calculate $\xi_{\text {pair }}$, defined as $[21,10]$ :

$$
\xi_{\text {pair }}=\sqrt{\frac{\int d \mathbf{r} g(\mathbf{r}) \mathbf{r}^{2}}{\int d \mathbf{r} g(\mathbf{r})}}=\sqrt{\frac{\int d \mathbf{k}\left[\partial_{\mathbf{k}} \phi(\mathbf{k})\right]^{2}}{\int d \mathbf{k} \phi^{2}(\mathbf{k})}}
$$

where

$$
g_{a}(\mathbf{r})=\frac{1}{n^{2}}\left|\left\langle\psi_{\uparrow}^{\dagger}(\mathbf{r}) \psi_{\downarrow}^{\dagger}(\mathbf{0})\right\rangle\right|^{2}
$$

and $\psi_{\sigma}(\mathbf{r})$ is the fermionic field operator, $\partial_{\mathbf{k}}$ is the gradient operator in $\mathbf{k}$-space, and $\phi(\mathbf{k})=\Delta_{\mathbf{k}} / E_{\mathbf{k}}\left(1-2 f_{F}\left(E_{\mathbf{k}}\right)\right.$ is the Fourier transform of $g(\mathbf{r})$. Here $\Delta(\mathbf{k})$ is the superconducting gap considered in the $d$-wave symmetry, $E_{\mathbf{k}} \equiv \sqrt{\xi_{\mathbf{k}}^{2}+\Delta_{\mathbf{k}}^{2}}$, with $\xi_{\mathbf{k}}$ the normal-state dispersion, and $f_{F}\left(E_{\mathbf{k}}\right)$ is the Fermi distribution. At zero temperature, $\xi_{\text {pair }}$ is infinite in the $d$-wave case, due to a logarithmically divergence at the numerator of (60) [10]. We will show that at finite temperature, the pair length is instead finite. As done in reference [10], let us consider the contribution of the numerator and of the denominator of equation (60) separately. Since we are interested in possible divergences, we can perform a nodal approximation, which consists in linearize the quasiparticle dispersion near the spectrum nodal points: $\xi_{\mathbf{k}} \simeq v_{F} k_{\|} \equiv v_{F} k_{x}, \Delta_{\mathbf{k}} \simeq v_{\Delta} k_{\perp} \equiv v_{\Delta} k_{y}$. In this way, we obtain for the numerator:

$$
\begin{aligned}
\mathcal{N}= & \frac{1}{N} \sum_{k_{x}, k_{y}}\left[\left[v_{F} \xi_{\mathbf{k}} \Delta_{\mathbf{k}}\left(\frac{2 f_{F}^{\prime}\left(E_{\mathbf{k}}\right)}{E_{\mathbf{k}}^{2}}+\frac{1-2 f_{F}\left(E_{\mathbf{k}}\right)}{E_{\mathbf{k}}^{3}}\right)\right]^{2}\right. \\
& \left.+\left[v_{\Delta}\left(\frac{2 f_{F}^{\prime}\left(E_{\mathbf{k}}\right)}{E_{\mathbf{k}}^{2}} \xi_{\mathbf{k}}^{2}-\frac{1-2 f_{F}\left(E_{\mathbf{k}}\right)}{E_{\mathbf{k}}^{3}} \Delta_{\mathbf{k}}^{2}\right)\right]^{2}\right]
\end{aligned}
$$

and for the denominator:

$$
\mathcal{D}=\frac{1}{N} \sum_{k_{x}, k_{y}}\left[\frac{\Delta_{\mathbf{k}}}{E_{\mathbf{k}}}\left(1-2 f_{F}\left(E_{\mathbf{k}}\right)\right)\right]^{2}
$$

where $f_{F}$ is the Fermi distribution and $f_{F}^{\prime}$ its first derivative. Following reference [10], we introduce the polar coordinates $(E, \theta)$, such that near a node we have $\xi_{\mathbf{k}}=E \cos \theta$ and $\Delta_{\mathbf{k}}=E \sin \theta$, and we usethe identity:

$$
\frac{1}{N_{s}} \sum_{k_{x} k_{y}}=\int \frac{d \theta d E E}{v_{F} v_{\Delta}}
$$

so that the equations (62) and (63) can be rewritten as:

$$
\begin{aligned}
\mathcal{N}= & \int \frac{d \theta d E}{v_{F} v_{\Delta}} E \\
& \times\left[\left[v_{F} \cos \theta \sin \theta\left(2 f_{F}^{\prime}(E)+\frac{1-2 f_{F}(E)}{E}\right)\right]^{2}\right. \\
& \left.+\left[v_{\Delta}\left(2 f_{F}^{\prime}(E) \cos \theta^{2}-\frac{1-2 f_{F}(E)}{E} \sin \theta^{2}\right)\right]^{2}\right]
\end{aligned}
$$


and

$$
\mathcal{D}=\int \frac{d \theta d E}{v_{F} v_{\Delta}} E \sin \theta\left(\tanh \frac{\beta E}{2}\right)^{2},
$$

respectively. Since the integration in the variable $E$ is carried from zero to a certain finite upper cutoff, the integral in (66) is finite. For the integral (65) we carry a Taylor expansion of the Fermi function around $E=0$ :

$$
\begin{aligned}
2 f_{F}^{\prime}(E) & +\frac{1-2 f_{F}(E)}{E}=2 f_{F}^{\prime}(0)+2 f_{F}^{\prime \prime}(0) E \\
& +\frac{1-2 f_{F}(0)-2 f_{F}^{\prime}(0) E-f_{F}^{\prime \prime}(0) E^{2}}{E}+O\left(E^{2}\right) \\
& \approx f_{F}^{\prime \prime}(0) E
\end{aligned}
$$

and

$$
\begin{aligned}
2 f_{F}^{\prime}(E) \cos \theta^{2} & -\frac{1-2 f_{F}(E)}{E} \sin \theta^{2}= \\
& \approx-2 f_{F}^{\prime}(0)-f_{F}^{\prime \prime}(0) E .
\end{aligned}
$$

Substituting (66) and (67) in (64), we obtain:

$$
\begin{aligned}
\mathcal{N} & =\int \frac{d \theta d E}{v_{F} v_{\Delta}} E\left[\left(v_{F} \cos \theta \sin \theta f_{F}^{\prime \prime}(0) E\right)^{2}\right. \\
& \left.+v_{\Delta}^{2}\left(2 f_{F}^{\prime}(0)+f_{F}^{\prime \prime}(0) E\right)^{2}\right]
\end{aligned}
$$

which is indeed a finite quantity. Moreover, introducing the adimensional variable $E^{\prime}=\beta E$ in (64) and (65) that the pair length $\xi_{\text {pair }}$ diverges in temperature as $1 / T$.

In summary, we have that the pair length (60) is a well defined quantity at $T \neq 0$ for the d-wave symmetry, at variance with the zero temperature case.

\section{References}

1. T. Timusk, B. Statt, Rep. Prog. Phys. 62, 61 (1999)

2. M. Randeria, Proceedings of the International School of Physics "Enrico Fermi", CXXXVI Course, Varenna (1997), arXiv: cond-Mat/9710223

3. Z.A. Xu, N.P. Ong, Y. Wang, T. Kakeshita, S. Uchida, Nature 406, 486 (2000); see also U. Thisted, J. Nyhus, T. Suzuki, J. Hori, K. Fossehim, arXiv: cond-mat/0304009

4. J.V. Emery, S.A. Kivelson, Nature 374, 434 (1998)

5. Q. Chen, I. Kosztin, B. Jankó, K. Levin, Phys. Rev. Lett. 81, 4708 (1998)

6. M. Fortes, M.A. Solís, M. de Llano, V.V. Tolmachev, Physica C 364-365, 95 (2001)
7. P. Noziéres, S. Schmitt-Rink, J. Low Temp. Phys. 59, 195 (1985)

8. Y.-J. Kao, A.P. Iyengar, Q. Chen, K. Levin, Phys. Rev. 64, 140505 (2001)

9. F. Pistolesi, G.C. Strinati, Phys. Rev. B 49, 6356 (1994)

10. L. Benfatto, A. Toschi, S. Caprara, C. Castellani, Phys. Rev. B 66, 054515 (2002)

11. R. Micnas, M.H. Pedersen, S. Schafroth, T. Schneider, J.J. Rodríguez-Núñez, H. Beck, Phys. Rev. B 52, 16223 (1995)

12. M. Yu. Kagan, R. Frésard, M. Capezzali, H. Beck, Phys. Rev. B 57, 5995 (1998)

13. J.M. Singer, M.H. Pedersen, T. Schneider, H. Beck, H.-G. Matuttis, Phys. Rev. B 54, 1286 (1996)

14. R. Micnas, J. Ranninger, S. Robaszkiewicz, Rev. Mod. Phys. 62, 113 (1990); T. Kostyrko, R. Micnas, Phys. Rev. B 46, 11025 (1992)

15. L.P. Kadanoff, G. Baym, Quantum Statistical Mechanics (New York, W.A. Benjamin Inc., 1962)

16. A. Sewer, H. Beck, Phys. Rev. B 64, 224524 (2001)

17. J.R. Engelbrecht, A. Nazarenko, M. Randeria, E. Dagotto, Phys. Rev. B 57, 13406 (1998)

18. N. Andrenacci, A. Perali, P. Pieri, G.C. Strinati, Phys. Rev. B 60, 12410 (1999)

19. See, for example: M. Greiner, O. Mandel, T. Esslinger, T.W. Hänsch, I. Bloch, Nature 415, 39 (2002); C. Orzel, A.K. Tuchman, M.L. Febnselau, M Yasuda, M.A. Kasevich, Science 291, 2386 (2001)

20. B. Paredes, J.I. Cirac, Phys. Rev. Lett. 90, 150402 (2003)

21. F. Pistolesi, G.C. Strinati, Phys. Rev. B 53, 15168 (1996)

22. D. Forster, Hydrodynamic Fluctuations, Broken Symmetry, and Correlation Functions (Benjamin, MA, 1975)

23. For a discussion on intrinsic vs. extrinsic pseudogap scenario, see, e.g., J. Stajic, A. Iyengar, K. Levin, B.R. Boyce, T.R. Lemberger, Phys. Rev. B 68, 0245020 (2003)

24. D. van der Marel, Optical signatures of electron correlations in the cuprates, Chapter in "Strong interactions in low dimensions", Series: Physics and Chemistry of Materials with Low-Dimensional Structures, Vol. 25, edited by D. Baeriswyl, L. Degiorgi (Kluwer, 2005), VI, ISBN: 1-4020-1798-7, cond-mat/0301506

25. A.A. Varlamov, G. Balestrino, E. Milani, D.V. Livanov, Adv. Phys. 48, 655 (1999)

26. A.A. Abrikosov, Fundamental of the Theory of Metals (Elsevier, Amsterdam, 1988), Chap. 17

27. G.-Q. Zheng, H. Ozaki, W.G. Clark, Y. Kitaoka, P. Kuhns, A.P. Reyes, W.G. Moulton, T. Kondo, Y. Shimakawa, Y. Kubo, Phys. Rev. Lett. 85, 405 (2000)

28. E. Altman, E. Demler, M.D. Lukin, Phys. Rev. A 70, $013603(2004)$ 